Commun. Math. Phys. 139, 103-139 (1991)

Communications in

Mathematical

Physics

(C) Springer-Verlag 1991

\title{
Boundary Conditions for Quantum Mechanics on Cones and Fields Around Cosmic Strings
}

\author{
Bernard S. Kay ${ }^{1}$ and Urban M. Studer ${ }^{2}$ \\ 1 Department of Applied Mathematics and Theoretical Physics, University of Cambridge, \\ 3, Silver Street, Cambridge CB3 9EW, UK \\ ${ }_{2}$ Institute for Theoretical Physics, ETH Hoenggerberg, CH-8093 Zurich, Switzerland
}

Received August 13, 1990; in revised form January 2, 1991

\begin{abstract}
We study the options for boundary conditions at the conical singularity for quantum mechanics on a two-dimensional cone with deficit angle $\leqq 2 \pi$ and for classical and quantum scalar fields propagating with a translationally invariant dynamics in the $1+3$ dimensional spacetime around an idealized straight infinitely long, infinitesimally thin cosmic string. The key to our analysis is the observation that minus-the-Laplacian on a cone possesses a one-parameter family of selfadjoint extensions. These may be labeled by a parameter $R$ with the dimensions of length - taking values in $[0, \infty)$. For $R=0$, the extension is positive. When $R \neq 0$ there is a bound state. Each of our problems has a range of possible dynamical evolutions corresponding to a range of allowed $R$-values. They correspond to either finite, for $R=0$, or logarithmically divergent, for $R \neq 0$, boundary conditions at zero radius. Non-zero $R$-values are a satisfactory replacement for the (mathematically ill-defined) notion of $\delta$-function potentials at the cone's apex.

We discuss the relevance of the various idealized dynamics to quantum mechanics on a cone with a rounded-off centre and field theory around a "true" string of finite thickness. Provided one is interested in effects at sufficiently large length scales, the "true" dynamics will depend on the details of the interaction of the wave function with the cone's centre (/field with the string etc.) only through a single parameter $R$ (its "scattering length") and will be well-approximated by the dynamics for the corresponding idealized problem with the same $R$-value. This turns out to be zero if the interaction with the centre is purely gravitational and minimally coupled, but non-zero values can be important to model nongravitational (or non-minimally coupled) interactions. Especially, we point out the relevance of non-zero $R$-values to electromagnetic waves around superconducting strings. We also briefly speculate on the relevance of the $R$-parameter in the application of quantum mechanics on cones to $1+2$ dimensional quantum gravity with massive scalars.
\end{abstract}




\section{Introduction}

Recently, there has been considerable interest in quantum theory on spacetimes with conical singularities. The reasons are (at least) twofold: Firstly, in $1+3$ dimensions, such spacetimes may be regarded as idealizations $[1,2]$ of the spacetimes around certain types of cosmic strings (in situations where the thickness of the strings may be neglected). Here the prototype spacetime is that due to the presence of an infinitely long static straight string in a flat spacetime and is represented by the manifold $\mathscr{M} \approx \mathbb{R} \times \mathbb{R}^{+} \times \mathbf{S} \times \mathbb{R}$ with metric

$$
g=d t^{2}-d \varrho^{2}-\kappa^{2} \varrho^{2} d \theta^{2}-d z^{2},
$$

where $\kappa$ (assumed to satisfy $0<\kappa \leqq 1$ ) is related to the linear mass density $\langle 1\rangle^{1} \lambda$ of the string by $\kappa=1-4 \lambda$, and corresponds to a deficit angle $\delta=2 \pi(1-\kappa)$ (see e.g. [3, $35-38,48,54,55]$ and also the review [1]). The prototype quantum field theory on such a spacetime is that of the covariant Klein-Gordon equation

$$
\left(\square_{g}+\mu^{2}\right) \phi=0 \text {. }
$$

Many authors have studied a ground state for this system (typically in the case $\mu=0)$ and calculated, in this state, the expectation value of the renormalised (typically conformally coupled) energy momentum tensor [4-10,38].

The computed effect is small; the energy density varies as the inverse fourth power of the distance from the string, and, in realistic models based on GUT scale strings, the integrated (i.e. up to a typical string radius) energy per unit length of string is only of the order of $10^{-8}$ of the energy per unit length of the string itself. Nevertheless, the computation is an interesting matter of principle (closely related to the Casimir effect $[11,12])$ and may be valuable as a preliminary exercise prior to tackling possibly more interesting questions such as pair-creation effects due to accelerated segments of string (cf. [10]).

Also of interest is the corresponding classical field theory of (1.2) which is related to physically important questions such as the scattering of electromagnetic waves by cosmic strings (see e.g. [51, 52]).

Secondly, in $1+2$ dimensions, it has recently been argued $[13,14]$ that the equation of relativistic

$$
i \frac{\partial \psi}{\partial t}=\left(\mu^{2}-\Delta\right)^{1 / 2} \psi
$$

or non-relativistic $\langle 2\rangle$

$$
i \frac{\partial \psi}{\partial t}=-\Delta \psi
$$

quantum mechanics where $\Delta$ is the Laplace Beltrami operator on the twodimensional cone $\mathscr{C} \approx \mathbb{R}^{+} \times \mathbf{S}$ with metric

$$
h=d \varrho^{2}+\kappa^{2} \varrho^{2} d \theta^{2}
$$

(we again assume $0<\kappa \leqq 1$ ) plays a key role in the solution of $1+2$ dimensional quantum gravity with massive scalar matter (at least in the regime of heavy masses and slow relative motion). Let us mention that quantum mechanics on a cone (1.4)

1 The numbers $\langle 1\rangle,\langle 2\rangle, \ldots$ refer to the section entitled Notes at the end of the paper 
has also recently been discussed [15] as an analogue for some aspects of the problem of canonical quantization of $1+3$ dimensional quantum gravity where one expects to have to deal with a wave functional defined on the superspace [16] of 3-geometries - an infinite-dimensional space with conical singularities.

In this paper, we wish to clarify the question of boundary conditions which, in all these problems, need to be imposed at the conical singularity - aiming, in each case, to describe the most general possible dynamical evolution consistent only with the very general principles (such as unitarity for Eq. (1.4)) which one expects to hold in a wide range of physical applications. Our essential result will be that each of our problems admits a one-parameter family of distinct dynamics corresponding to different possible boundary conditions. Of course, from a physical point of view, the problems we are posing either (in the application to cosmic strings) overidealize the situation being modelled, or (in the application to $1+2$ dimensional gravity) represent only a building block in a full theory. In particular, in the cosmic-string application, the very need for boundary conditions may be viewed as a result of overidealizing the string's thickness as strictly zero. In fact, in a more realistic model, the string would have a non-singular core of finite thickness and the dynamics of our field would be uniquely determined by the details of its interaction with this core. (Similarly, one can think of Eq. (1.4) as overidealizing some interaction - possibly non-minimally coupled or with the inclusion of a potential term - between the wave function and a rounded off cone.) However, what we particularly wish to emphasize here is that there is an intimate connection between the problem of boundary conditions for an idealized string and the (uniquely defined) dynamics on such a "true" string or such a "rounded-off cone." Indeed, as will be discussed further in a separate paper [42], the fact that our idealized dynamics is labelled by a single parameter may be interpreted as an indication that, provided one is interested in effects at sufficiently large lengthscales (i.e. sufficiently low energies) the dynamics on any given true string (or rounded-off cone) will depend, to a very good approximation, on the details of the core-interaction only through a single parameter. In the application to cosmic strings (or rounded-off cones) the one-parameter family of options for the dynamics of our various idealized problems will then be of physical relevance as providing candidates for the description of the large-scale behaviour of the dynamics on a true cosmic string (or rounded-off cone).

This use of our idealized problems to model the large-scale behaviour of field theory around true strings, or quantum mechanics on rounded-off cones will be treated in Sect. 5 of the present paper, and discussed further later in this introduction. However, our treatment of the idealized models in the first four sections of the paper is not tied to this particular application. For example, in Sect. 2, we shall also briefly consider the application to $1+2$ dimensional quantum gravity, speculating that the parameter $R$ may correspond to a new parameter needed over and above the mass in determining the interaction of each species of massive scalar particle with gravity.

First, we turn to an outline of our results for the idealized problems. We shall actually isolate four distinct problems (A-D below) - all interrelated, but each requiring a separate discussion.

(A) Firstly for the non-relativistic quantum mechanics on a cone (1.4), the standard ideology (see e.g. $[17,18]$ ) reduces the boundary-condition problem to the study of the possible self-adjoint extensions of (minus) the Laplacian, $-\Delta$, 
when it is defined on the domain of smooth functions compactly supported away from the conical singularity in the Hilbert space of square-integrable functions on a two dimensional cone. In coordinates, this amounts to studying

$$
-\Delta=-\left(\frac{\partial^{2}}{\partial \varrho^{2}}+\frac{1}{\varrho} \frac{\partial}{\partial \varrho}+\frac{1}{\kappa^{2} \varrho^{2}} \frac{\partial^{2}}{\partial \theta^{2}}\right)
$$

on $\langle 3\rangle$

$$
C_{0}^{\infty}\left(\mathbb{R}^{+} \times \mathbf{S}\right) C L^{2}\left(\mathbb{R}^{+} \times \mathbf{S}, \varrho d \varrho d \theta\right) .
$$

In Appendix 1, we show that this symmetric operator has (for $0<\kappa \leqq 1$ ) a oneparameter family of self-adjoint extensions which we label

$$
\left\{-\Delta^{R}: R \in[0, \infty)\right\},
$$

each corresponding to a different choice of boundary condition at the conical singularity. One of these, the extension for $R=0$ (the Friedrichs extension [18]), $-\Delta^{0}$, is a positive operator. It corresponds to wave functions which are regular at the conical singularity. Each of the others has a bound state with eigenvalue given by $-q^{2}$, where $\langle 4\rangle$

$$
q=2 e^{-c} R^{-1}, \quad C=\text { Euler's constant } .
$$

They correspond to different strengths of logarithmic divergence

$$
\psi \sim \text { const } \ln (\varrho / R)
$$

of the wave function near the conical singularity. In Sect. 1, we point out that each choice of parameter $R$ will provide an inequivalent possible quantum dynamics and begin the discussion (which will be continued in Sect. 5) of their significance. We also describe how, to each choice, corresponds a different eigenfunction expansion for the Laplacian - in terms of Bessel functions of the first kind for the Friedrichs extension and of combinations of Bessel functions of the first and second kind, together with the above bound state, in all other cases.

(B) Secondly, we point out, still in Sect. 2, that in the case of the relativistic oneparticle dynamics on the cone (1.3), one obtains different acceptable timeevolutions for each self-adjoint extension, $-\Delta^{R}$ of the Laplacian for which $\mu^{2}-\Delta^{R}$ turns out to be a positive operator. In terms of $q$ (see (1.7) and Note $\langle 4\rangle$ ) this requires $0 \leqq q \leqq \mu$, and in the special case of zero mass, fixes the extension uniquely to be the Friedrichs extension.

(C) The third problem we discuss is that of boundary conditions for the classical Klein-Gordon Eq. (1.2) on the spacetime around an idealized cosmic string $(\mathscr{M}, \mathrm{g})$ (1.1). Our main conclusion is that, to each self-adjoint extension $-\Delta^{R}, R \in[0, \infty)$ of minus the two-dimensional cone Laplacian, will correspond a different possible dynamical evolution - each corresponding to a different translationally invariant choice of boundary conditions at the string. We shall argue that, for the zero mass wave equation, only the dynamics corresponding to the Friedrichs extension $(R=0)$ can be stable. However, for non-zero mass, the range of extensions with $0 \leqq q \leqq \mu$ (with the possible exception of borderline cases - cf. Note $\langle 18\rangle)$ will be stable.

In an appendix (Sect. A2) we discuss more critically what the necessary ingredients are for an acceptable dynamical evolution for the classical Klein 
Gordon equation on a string and arrive at the notion of a (translationally invariant) "global symplectic extension" of the local dynamics. We check that all the candidate dynamical evolutions constructed in Sect. 3 satisfy this notion - but leave open the possible occurrence of further "exotic" possibilities. We shall however ignore this issue in the rest of the paper.

(D) Fourthly, we discuss in Sect. 4, the options for (translationally invariant) quantizations of the Klein-Gordon equation around an idealized cosmic string. We find that, to every option for the classical time evolution (and hence, as explained in Sect. 3, to every self-adjoint extension $\Delta^{R}$ of the Laplacian on a twodimensional cone) there corresponds an acceptable quantum dynamics in the sense that one obtains a well-defined field-algebra admitting a time-evolution automorphism. However, we show that only those dynamics which were classically stable (i.e. - modulo issues raised in Note $\langle 18\rangle-$ for which $0 \leqq q \leqq \mu$ ) can lead to quantizations which admit ground states. This is of course expected since the existence of a quantum-field-theoretic ground state is intimately related to the existence of a suitable one-particle dynamics for the analogue of (1.3) on our $1+3$ dimensional cosmic string exterior. (As for (1.3), this requires $0 \leqq q \leqq \mu$.) We conclude that in the case of a zero mass scalar quantum field around our idealized cosmic string, there is a unique $\langle 5\rangle$ quantization admitting a ground state. This corresponds to the choice of the Friedrichs extension, $-\Delta^{0}$, of minus the twodimensional cone Laplacian and coincides with the quantization considered by other authors (see [4-10, 38]). However, for a non-zero-mass field, there are other mathematical possibilities with $0 \leqq q \leqq \mu$.

To summarize, we find that, in each of the idealized situations (A-D) described above, the different possible boundary conditions correspond to different possible self-adjoint extensions $-\Delta^{R}$ of minus the Laplacian on the cone. For some of the problems, the full range, $R \in[0, \infty)$ of $R$-values is possible. For others, $R$ must be further restricted.

We now briefly discuss the significance of the different possible $R$-values, and attempt to relate our work to the existing literature on related topics. It is clear that, in several respects, the value $R=0$ is a distinguished value: It is the only value for which $-\Delta^{R}$ is a positive operator. It is also the only value for which the domain of $-\Delta^{R}$ consists of continuous functions which take finite values at the conical singularity. (We shall make this remark precise in the sequel.) Much of the existing work on spacetimes with conical singularities - be it concerned with scalar quantum mechanics on cones $[13,14]$ (see also [31]), be it with scalar quantum field theory around idealized cosmic strings [4-10] in fact - explicitly or implicitly - regards this latter finiteness or "regularity" condition as fundamental, and in this way, all the just-cited papers arrive uniquely at the $R=0$ boundary condition, and fail to consider the possibilities with $R \neq 0$. (We remark however that the work in [15] has recently [60] been extended to take into account the possible importance of non-Friedrichs extensions.)

Actually, if one takes the point of view that $R=0$ is a distinguished value, it is natural to identify the operators $-\Delta^{R}$ for $R$ other than zero as corresponding, in some sense, to perturbing $-\Delta^{R}$ by a $\delta$-function potential at the apex of the cone. (Cf. e.g. [44] where a similar remark is made.) However, we stress that there are mathematical difficulties with the notion of $\delta$-function potentials in more than one dimension, and, if one attempts to make sense of them, one is essentially forced back to consider the operators $-\Delta^{R}$ ! Note that these are difficulties which persist - 
and must be resolved in an analogous way - even when the deficit angle of the cone is zero, i.e. for point-interactions in ordinary two-dimensional Euclidean space. This, and related topics are extensively discussed in the work of Albeverio et al. [28] (see also [59]) which has many points of contact with the point of view of the present paper. In view of these difficulties, it seems to us preferable to regard the case $R=0$ as no more fundamental than any other $R$-value, but rather to think of all $R$-values as a priori on an equal footing, without prejudging the issue of which $R$-value is most appropriate in which application. Indeed, the fact that $R=0$ corresponds to regular wavefunctions is perhaps not as significant as it might at first appear: Even though - say in the case of a string with finite thickness - the true problem one is idealizing will have regular wave-functions, it is not sound to argue that this is therefore best modelled by regular wave-functions when one idealizes the string as having zero thickness. This is just one of several "pitfalls" that we shall discuss in Note $\langle 22\rangle$ to Sect. 5.2. (see also [42]) and we shall see below that extensions with non-zero $R$ are indeed often relevant to modelling such true dynamics.

Another advantage of regarding all $R$-values as on an equal footing is that this point of view ought to generalize from the scalar case considered here to the study of higher spin and gauge fields (including linearized gravitational perturbations) propagating on cones and around cosmic strings. In these cases, the first step would still be the analysis of an appropriate self-adjoint extension problem which, in the general case (and, after a suitable gauge fixing procedure in the case of gauge fields) would now concern a system of differential operators. In this connection, it is interesting to note that De Sousa Gerbert and Jackiw [33] have found that in the case of the Dirac equation on a "spinning cone" there is a one-parameter family of self-adjoint extensions none of which correspond to regular boundary conditions at the conical singularity. (It should be remarked that, as these authors explain, this spinning cone problem involves indefinite inner product spaces, and thus a suitably generalized notion of self-adjointness is required here.)

Finally, we return to the crucial question of the physical relevance of our discussion of the idealized problems $(A-D)$ to the problem of wavefunctions on a cone with a rounded-off apex or of (classical or quantum) fields propagating in the neighbourhood of a true cosmic string of finite thickness. As we indicated at the outset, it is natural to make the hypothesis that, for any specific model, say, for the true interaction of the cosmic-string interior with our dynamical field (or of a rounded-off cone - here we include a possible potential or non-minimal coupling term - with our non-relativistic wave function) there would be a particular choice of boundary conditions - i.e. a particular $R$-value - for the idealized problem which leads to a good approximation to the true dynamics. In fact there seems to be a very general relationship of this type - valid in a wide range of physical situations - between the possible large-scale effects of a small object and the options for defining the dynamics for the corresponding idealized system where the object is idealized as having zero size. A fuller discussion of this relationship, in a rather general setting, will be given elsewhere [42] (see also [43]). In Sect. 5, we shall discuss the extent to which this hypothesis is borne out for the specific systems (true string, and rounded-off cone) under discussion here. The main idea is that the $R$-value in the idealized problem should be chosen to equal the scattering length for the true interaction. Here, we refer to a two-dimensional analogue, which we introduce, to the familiar three dimensional notion of scattering length $\langle 6\rangle$. This idea should be of practical importance when one wants to calculate the extent to 
which the details of the dynamics inside the core of a string (or inside the roundedoff region of a cone) influence various physical effects. It suggests that - with the important proviso that one is interested in effects at sufficiently large length scales - the details of the interaction with the core should be well taken into account by a single parameter (namely the scattering length).

Section 5.1 states our principal result, which is that, in the case of minimally coupled quantum mechanics on a purely rounded-off cone, i.e.

$$
i \frac{\partial \psi}{\partial t}=-\Delta_{\tilde{h}} \psi
$$

$\left(\Delta_{\tilde{h}}\right.$ here denotes the Laplace-Beltrami operator for some smooth metric which coincides with (1.1) outside some small circle of constant $\varrho$ ) the scattering length is zero. (A similar result holds in the case of a classical Klein-Gordon field which interacts minimally only with the gravitational field in some true cosmic string interior). On the other hand, the addition of a potential term or non-minimal coupling term (supported inside the small circle) in Eq. (1.9)

$$
i \frac{\partial \psi}{\partial t}=-\left(\Delta_{\tilde{h}}+\tilde{V}\right) \psi
$$

or of some non-minimal or non-gravitational interaction with the field in the interior of the cosmic string, leads to a non-zero scattering length. Such cases might heuristically be modelled by the addition of a $\delta$-function potential (see above) to $-\Delta^{0}$. However, in such an approach, one would discover that the strength of the required $\delta$-function turns out to be ill-defined, requiring an infinite renormalization. Our point of view has the advantage that the scattering length $R$ is a physically meaningful finite quantity which may be calculated for any given $\tilde{V}$.

In Sect. 5.2, we point out a number of subtleties in the relationship between the true and idealized problems. In particular we discuss the case of quantum mechanics, or a classical Klein Gordon field, with a strong positive potential of small support. This is of importance as a model for electromagnetic waves propagating around a superconducting cosmic string [52] $\langle 7\rangle$. We explain why, for this problem, the scattering length, while small, should not be approximated by zero. We also caution that, when one is using our idealized models to approximate the large-scale behaviour of true cosmic strings and rounded-off cones, those properties of the idealized dynamics which have to do with small scales will not be physically relevant (in the language of [42], not "believable"). Such a property is the existence of a bound state for small non-zero values of $R$ (large values of $q$ ) and the consequent classical and quantum instabilities mentioned in paragraphs $\mathrm{C}$ and $\mathrm{D}$ above. In consequence, in the scalar analogue for electromagnetic waves around superconducting strings just mentioned, these classical and quantum instabilities in the idealized model should be regarded as spurious as far as the true situation being approximated is concerned. Furthermore, we shall explain how the true quantum vacuum state can be better approximated at large scales by a "modified vacuum state" with a non-zero $R$-value than by the $R=0$ vacuum, in spite of the fact (cf. paragraph D above) that the latter is the only vacuum state which exists when one quantizes modes of all length scales so as to obey the exact idealized dynamics. At the very end of Sect. 5.2, we return to the question of the ground state expectation value of the renormalized energy-momentum tensor for a quantum field in the neighbourhood of a cosmic 
string. We point out (1) that, for an idealized string, one can, in principle, calculate the expectation value of the energy-momentum tensor in any of the modified vacuum states just mentioned (i.e. for any $R$-value). (2) For a given true string, it is a non-trivial question, which will require further investigation, to decide whether the result of any of these calculations will well-approximate the true vacuum expectation value.

To end this introduction, we mention two issues, which, while closely related to the topics of this paper, will not be discussed here. Firstly, we do not consider the case of a charged scalar field which interacts with an idealized cosmic string carrying a non-zero magnetic flux. In this case, one would have to take into account not only the different possible boundary conditions at the string but also the non-trivial Aharonov-Bohm [50] magnetic potential. For the scalar case with boundary conditions which generalize the case $R=0$ of the present paper, this problem has been discussed by Dowker (see $[8,9])$. For the Dirac case, see [61] where the relevant self-adjointness extension problem is discussed. (Note also that the $1+2$ dimensional quantum mechanical analogue to this Aharonov Bohm problem is related to (see $[33,61]$ ) de Sousa Gerbert and Jackiw's problem of quantum mechanics on a "spinning cone" which we mentioned above.) It might be interesting to try to give a unified treatment of the Aharonov Bohm problem in which the (appropriate generalization of the) $R$-parameter discussed in the present paper and the Aharonov-Bohm parameter (i.e. charge multiplied by magnetic flux modulo $2 \pi$ ) appear on the same footing. In this connection, the recent interesting ideas of Landsman [49] on a class of problems which includes the AharonovBohm situation may be relevant.

A second problem which we do not treat, but which may be interesting to explore is how the different choices of boundary conditions isolated in this paper would arise in a path-integral approach.

\section{Quantum Mechanics on a Two-Dimensional Cone}

As we explain in more detail and prove in Appendix 1, minus the Laplacian on the cone, i.e.

$$
-\Delta=-\left(\frac{\partial^{2}}{\partial \varrho^{2}}+\frac{1}{\varrho} \frac{\partial}{\partial \varrho}+\frac{1}{\kappa^{2} \varrho^{2}} \frac{\partial^{2}}{\partial \theta^{2}}\right)
$$

on $\langle 3\rangle$

$$
C_{0}^{\infty}\left(\mathbb{R}^{+} \times \mathbf{S}\right) \subset L^{2}\left(\mathbb{R}^{+} \times \mathbf{S}, \varrho d \varrho d \theta\right)
$$

has (for $0<\kappa \leqq 1)\langle 8\rangle$ a one-parameter family of self-adjoint extensions

$$
\left\{-\Delta^{R}: R \in[0, \infty)\right\} \text {. }
$$

In fact, on each $m$-sector consisting of functions of form $f_{m}(\varrho) e^{i m \theta},(1.6)$ acts as the ordinary differential operator

$$
-\Delta_{m}=-\frac{\partial^{2}}{\partial \varrho^{2}}-\frac{1}{\varrho} \frac{\partial}{\partial \varrho}+\frac{m^{2}}{\kappa^{2} \varrho^{2}} .
$$

For $m \neq 0$, each of these operators is essentially self-adjoint on the domain $C_{0}^{\infty}\left(\mathbb{R}^{+}, \varrho d \varrho\right) \subset L^{2}\left(\mathbb{R}^{+}, \varrho d \varrho\right)$. However, in the cylindrically symmetric sector $m=0$, 
it turns out that there is a one-parameter family of self-adjoint extensions, $\left\{-\Delta_{0}^{R}, R \in[0, \infty)\right\}$ characterized by the following boundary conditions at $\varrho=0$ :

$$
\begin{aligned}
& \lim _{\varrho \rightarrow 0}\left([\ln (\varrho / R) \varrho d / d \varrho-1] f_{0}\right)(\varrho)=0, \text { for } R \in(0, \infty), \\
& \lim _{\varrho \rightarrow 0}\left(\varrho d / d \varrho f_{0}\right)(\varrho)=0, \text { for } R=0 .
\end{aligned}
$$

(Note that (2.3) is just a more precise form of (1.8).) The case $R=0$ corresponds to the Friedrichs extension [18] of (1.6), $-\Delta^{0}$, which is a positive operator with continuous spectrum. In this case, the set of functions

$$
\left\{\psi_{m, p}(\varrho, \theta)=(2 \pi)^{-1 / 2} J_{|m / \kappa|}(p \varrho) e^{i m \theta}: p \in \mathbb{R}^{+}, m \in \mathbb{Z}\right\}
$$

$\left(J_{v}\right.$ denotes a Bessel function of the first kind [32]) constitute a "complete set of (generalized) eigenfunctions" with eigenvalues $p^{2}$ and continuum normalization

$$
\int_{0}^{\infty} \int_{0}^{2 \pi} \psi_{m, p}(\varrho, \theta)^{*} \psi_{m^{\prime}, p^{\prime}}(\varrho, \theta) \varrho d \varrho d \theta=p^{-1} \delta\left(p-p^{\prime}\right) \delta_{m, m^{\prime}} .
$$

For all other values of $R,-\Delta^{0}$ fails to be positive $\langle 9\rangle$, acquiring the bound state

$$
\psi_{\text {bound }}^{(q)}(\varrho)=\pi^{-1 / 2} q K_{0}(q \varrho)
$$

( $K_{0}$ is the appropriate Bessel function of the $3 r d$ kind [32]) with eigenvalue $-q^{2}$, where

$$
q=2 e^{-c} R^{-1}, \quad C=\text { Euler's constant. }
$$

Also the continuum eigenfunctions in the $m=0$ sector must be replaced by the linear combinations of Bessel functions of the first and second kinds

$$
\psi_{0, p}^{(q)}(\varrho)=(2 \pi)^{-1 / 2}\left[\beta^{2}(p)+1\right]^{-1 / 2}\left(J_{0}(p \varrho)+\beta(p) N_{0}(p \varrho)\right),
$$

where

$$
\beta(p)=\frac{\pi}{2}\left(\ln \left[\frac{q}{p}\right]\right)^{-1}
$$

with eigenvalue $p^{2}$ and continuum normalization analogous to (2.5). Relabelling our self-adjoint extensions by using as parameter $q$ in place of $R\langle 4\rangle$, a complete set of eigenfunctions for $-\Delta^{(q)}, q>0$ thus consists of

$$
\left\{\psi_{\text {bound }}^{(q)}\right\} \cup\left\{\psi_{0, p}^{(q)}: p \in \mathbb{R}^{+}\right\} \cup\left\{\psi_{m, p}: m \neq 0, p \in \mathbb{R}^{+}\right\},
$$

where the three terms in the union are defined by (2.6), (2.7), and (2.4) respectively.

Standard arguments based on Stone's theorem and the spectral theorem (see e.g. [17]) lead us to regard each of the self-adjoint extensions, $-\Delta^{(q)}, q \in[0, \infty)$, as providing an acceptable integration of the Schrödinger equation on the cone (1.4) and hence implicitly as providing an acceptable choice of boundary conditions $\langle 10\rangle$. We may use the spectral Theorem [17] to define, for each $q$, the timeevolution operator

$$
U^{(q)}(t)=\exp \left(i \Delta^{(q)} t\right) .
$$

For comparison with later developments (cf. Sects. 3 and A2) it is worth recalling the two reasons why each of these (distinct) time-evolutions may be viewed as acceptable: First, for each $q, U^{(q)}(t)$ provides a formal solution to (1.4). 
Second, $U^{(q)}(t)$ preserves the $L^{2}$-inner product

$$
\left\langle\psi_{1} \mid \psi_{2}\right\rangle=\int \psi_{1}^{*} \psi_{2} \varrho d \varrho d \theta
$$

(it is unitary). Thus we conclude that quantum mechanics on the cone should actually be labelled by a new parameter, $q$, which tells us which self-adjoint extension is selected.

In the relativistic case $\langle 11\rangle$, to make sense of the operator $\left(\mu^{2}-\Delta\right)^{1 / 2}$, one must restrict attention either to the case $q=0$, where there is no bound state, or to $q$ in the range

$$
0<q \leqq \mu
$$

so that $\mu^{2}-q^{2}$ and hence $\mu^{2}-\Delta^{(q)}$ is positive. Note that for $\mu=0$, one is obliged to take the Friedrichs extension, $-\Delta^{(0)}$. (See however Sect. 5.)

All computed quantities, for example, the propagator

$$
\text { “ }\left\langle\delta\left(x_{2}\right)\left|\exp \left(i \Delta^{(q)} t\right)\right| \delta\left(x_{1}\right)\right\rangle "
$$

will of course depend on the value of $q$. We remark that the propagator calculated in [14] (cf. also [31] and references therein) for the non-relativistic case is now seen to correspond to the choice of $q=0$ (the Friedrichs extension) but one could equally well use the set of eigenfunctions (2.9) to compute (2.12) for any other value of $q$.

Two considerations are of general importance in assessing the possible relevance of the different self-adjoint extensions in different physical interpretations for quantum mechanics on a cone. First, we remark that, in the case of $\kappa=1$ (zero deficit angle) where $-\Delta$ on $C_{0}^{\infty}\left(\mathbb{R}^{+} \times \mathbb{S}\right) \subset L^{2}\left(\mathbb{R}^{+} \times \mathbb{S}\right.$, $\left.\varrho d \varrho d \theta\right)$ may be regarded as minus-the-Laplacian on flat two-dimensional space with the origin removed, the Friedrichs extension $(q=0)$ is just minus the usual Laplacian on flat two-dimensional space (i.e. the unique self-adjoint extension of $-\Delta$ on $C_{0}^{\infty}\left(\mathbb{R}^{2}\right)$ $\left.C L^{2}\left(\mathbb{R}^{2}\right)\langle 12\rangle\right)$. The extensions for $q>0$, on the other hand, may be interpreted as describing quantum mechanics on flat two-dimensional space with "non-trivial physics" supported at a point - or on flat three-dimensional space with translationally invariant "non-trivial physics" supported on a line $\langle 13\rangle$.

Our second general consideration is that, in any physical interpretation in which minus-the-Laplacian on a cone is taken to represent a limiting case of minus-the-Laplace-Beltrami operator on a smooth (complete) Riemannian manifold in which the conical singularity is "rounded-off" (with no additional nonminimal coupling, or potential term) then one expects only the Friedrichs extension $(q=0)$ to be relevant. On the other hand, if one is using minus the Laplacian on a cone to represent a limiting case of Eq. (1.10) where one additionally has a potential term $\widetilde{V}$ or (if $\widetilde{V}$ represents some multiple of the Riemann scalar) non-minimal coupling term, then it turns out that non-zero $q$-values are relevant. However, we shall postpone a discussion of the sense in which this is true, and the reason why it is true to Sect. 5.

It is natural to ask what relevance, if any, the extensions with $q>0$ may have in the application to $1+2$ dimensional quantum gravity with (say) one species of real scalar particles $[13,14]$. This is usually expected to be labelled by a single parameter - the particle mass. However, we speculate that it may actually sit inside a richer two-parameter family of theories - labelled by both the mass and the $q$-value. $q$ would represent a new attribute of each species of scalar particle, needed, 
in addition to its mass, to specify its interaction with gravity. In view of our two general remarks above, the case of $q=0$ would then be interpretable as corresponding to particles which interact "purely minimally gravitationally" while other $q$-values would correspond to particles which also have a non-trivial zerorange interaction or non-minimal coupling.

Assuming that our model is physically realizable, one should be able to measure the value of $q$ which is physically selected by measuring the "phase shift" for the scattering $\langle 14\rangle$ of $m=0$ sector waves as a function of energy. For the Friedrichs extension $q=0$, this is energy-independent. For general $q$, one may use the standard asymptotic formulae for Bessel functions [32] to easily see that the eigenfunctions (2.7) behave for $\varrho \rightarrow \infty$ as

$$
\begin{gathered}
\psi_{0, p}^{(q)} \sim(2 \pi)^{-1}\left(\left(1+\beta^{2}(p)\right) p \varrho\right)^{-1 / 2}\left((1-i \beta(p)) e^{i(p \varrho-\pi / 4)}+(1+i \beta(p)) e^{-i(p \varrho-\pi / 4)}\right) \\
\left(\sim \text { indicates terms of order } \varrho^{-1} \text { are omitted }\right),
\end{gathered}
$$

from which we may read off that the "change in the phase shift" (defined to be zero for $q=0$ - see Note $\langle 14\rangle)$ is $\gamma(p)$, where

$$
\exp (2 i \gamma(p))=(1+i \beta(p))^{-1}(1-i \beta(p)) \text {. }
$$

Explicitly

$$
\gamma(p)=\pi-\operatorname{arcctg}\left(\frac{2}{\pi} \ln \left[\frac{q}{p}\right]\right)
$$

(where the arcctg is defined conventionally, taking values between 0 and $\pi$ ).

One may read off from this formula that, for non-zero values of $q, \gamma(p)$ starts at the positive value of $\pi$ at $p=0$ and decreases towards zero for large values of $p$. (Thus Levinson's Theorem $[39,40]$ holds $\langle 15\rangle$.) Note that the formula $(2.15)$ depends on the value of $q$ but not on the deficit angle of the cone (i.e. not on $\kappa$ ).

In the special case of zero deficit angle $(\kappa=1)$ the same asymptotic properties of Bessel functions easily lead to the formula

$$
\left.2 \pi\left|f_{p}\right|^{2}=\pi^{2} p^{-1}(\ln [q / p])^{2}+\pi^{2} / 4\right)^{-1}
$$

for the total cross-sectional length for scattering by a point interaction which corresponds to the boundary condition (2.3) (where $q$ is related to $R$ by $(1.7)$ ). In fact the scattering amplitude, $f_{p}$ in (2.16) is determined from the obvious twodimensional time-independent-scattering-theory ansatz

$$
\psi \sim \exp (\text { ip } \cdot \mathbf{x})+f_{p}(\theta) \exp (\text { ip } \varrho) / \varrho^{1 / 2}
$$

when one imposes the boundary condition $(1.8) /(2.3)$ on $\psi$. (The resulting $f_{p}(\theta)$ of course turns out to be independent of $\theta$.)

To end this section, we remark that the above discussion by no means exhausts all the possible ramifications of the topic of quantum mechanics on cones. In particular, we draw attention to Notes $\langle 10\rangle$ and $\langle 16\rangle$ which indicate two different directions in which it might be appropriate to extend the above mathematical framework. Note $\langle 10\rangle$ is concerned with modelling physical situations which involve the possibility of absorption of the wave function at the conical singularity. Note $\langle 16\rangle$ draws attention to the possibility that, depending on the physical application, it might be correct to include in the quantum Hamiltonian a potential term representing the gravitational and/or electromagnetic "self-force" experienced by a classical particle moving on a cone. (Other directions for generalization 
include consideration of "spinning cones" [33] as well as the related Aharonov Bohm problem [61] as we mentioned at the end of the introduction.)

\section{The Classical Klein-Gordon Equation Around an Idealized Cosmic String}

In this section, we discuss the mathematical options $\langle 10\rangle$ available for integrating the classical Klein-Gordon equation (1.2) on the $1+3$ dimensional spacetime $\mathscr{M}$ with metric $g$ (1.1) which represents the locally flat region around an infinite straight cosmic string in the idealization of infinitesimal thinness. The relevance of our results for a "true" cosmic string will be discussed in Sect. 5.

We treat the case of a real scalar field $\phi$. It is convenient to think of the problem in terms of the first-order form equations for Cauchy data $\varphi=\phi$ and $\pi=\partial \phi / \partial t$,

$$
\frac{\partial}{\partial t}\left(\begin{array}{l}
\varphi \\
\pi
\end{array}\right)=-\left(\begin{array}{rr}
0 & -1 \\
A_{\mu} & 0
\end{array}\right)\left(\begin{array}{l}
\varphi \\
\pi
\end{array}\right)
$$

with

$$
A_{\mu}=\mu^{2}-\Delta-\partial^{2} / \partial z^{2},
$$

where $\Delta$ is the Laplacian on a two dimensional cone, and we view the string's spatial geometry as the product of this two-dimensional cone and the real line.

Clearly the support of smooth Cauchy data, initially compactly supported away from the string, can (with a maximum speed of 1) eventually reach the "string" (i.e. the conical singularity) at $\varrho=0$ where it will require a boundary condition to tell it how to evolve further. Our aim is to find all possible options for this further evolution subject only to the requirement that the boundary conditions to which they correspond respect the translational invariance of the problem in the $z$-direction "along the string."

As might be expected, this problem is at least partially solved by means of the same family of self-adjoint extensions $-\Delta^{(q)}, q \in[0, \infty)$, which solved the analogous problem for the Schrödinger equation in Sect. 2. For each $\mu$ and $q$, let $A_{\mu}^{(q)}$ be the self-adjoint extension $\langle 17\rangle$

$$
A_{\mu}^{(q)}=\mu^{2}-\Delta^{(q)}-\partial^{2} / \partial z^{2}
$$

of $A_{\mu}$ on the real Hilbert space

$$
\mathscr{H}=L^{2}\left(\mathbb{R}^{+} \times \mathbf{S} \times \mathbb{R}, \varrho d \varrho d \theta d z\right)
$$

and extend the space of time- $t$ Cauchy data $\left(\varphi_{t}, \pi_{t}\right)=(\phi(t), \dot{\phi}(t))$ from $C_{0}^{\infty}\left(\mathbb{R}^{+} \times \mathbb{S}\right.$ $\times \mathbb{R}) \times C_{0}^{\infty}\left(\mathbb{R}^{+} \times \mathbf{S} \times \mathbb{R}\right)$ to the domain

$$
D_{\mu}^{(q)}=\mathscr{D}\left(\left|A_{\mu}^{(q)}\right|^{1 / 2}\right) \oplus \mathscr{H}
$$

in $\mathscr{H} \oplus \mathscr{H}$. Then it would appear that the global time-evolution

$$
\mathscr{T}_{\mu}^{(q)}(t):\left(\varphi_{0}, \pi_{0}\right) \rightarrow\left(\varphi_{t}, \pi_{t}\right), \quad t \in \mathbb{R}
$$

mapping $D_{\mu}^{(q)} \rightarrow D_{\mu}^{(q)}$ according to

$$
\mathscr{T}_{\mu}^{(q)}(t)=\left(\begin{array}{cc}
\cos \left(A_{\mu}^{(q)^{1 / 2}} t\right) & A_{\mu}^{(q))^{-1 / 2}} \sin \left(A_{\mu}^{(q)^{1 / 2}} t\right) \\
-A_{\mu}^{(q)^{1 / 2}} \sin \left(A_{\mu}^{(q)^{1 / 2}} t\right) & \cos \left(A_{\mu}^{(q)^{1 / 2}} t\right)
\end{array}\right)
$$


(where the functions in (3.6) are defined via the usual functional calculus using the spectral theorem [17]) is an acceptable solution to our problem. Note that due to the addition of the $-\partial^{2} / \partial z^{2}$ in (3.3), the spectrum of $A_{\mu}^{(q)}$ will (in contrast to that of $-\Delta^{(q)}$ ) now always be purely continuous. In the case $q=0$, it will be supported in the interval $\left[\mu^{2}, \infty\right)$ and otherwise in $\left[\mu^{2}-q^{2}, \infty\right)(\operatorname{see}(2.7))$. Note also that the case $q=0$ where one takes the Friedrichs extension [18] of $-\Delta$ clearly also corresponds to the Friedrichs extension of $A_{\mu}$.

We emphasize that $A_{\mu}^{(q)}$ is not in general positive. Nevertheless (3.6) will still make sense since, in spite of their appearance, the functions appearing in (3.6) clearly do not depend on any particular square root operation. The fact that $A_{\mu}^{(q)}$ is bounded below suffices for $D_{\mu}^{(q)}$ to be an invariant (dense) domain.

Formally, $\mathscr{T}_{\mu}^{(q)}(t)$ clearly solves the first order form Eqs. (3.1) with the boundary condition (2.3).

It is an interesting question to set up a suitable precise ideology for what one means by an "acceptable global time evolution" for this problem and to check that the above construction actually fulfills this ideology. We sketch how this can be done in Sect. A2. We recall that, in the case of the Schrödinger equation dealt with in Sect. 2, we relied on the familiar ideology - based on Stone's theorem and the spectral theorem (see e.g. $[17,18]$ ) - to immediately reduce the problem to a selfadjoint extension problem. For the present problem, it is not so evident whether Hilbert-space methods are always appropriate, since the natural conserved product for the Klein-Gordon equation is no longer an $L^{2}$-inner product (cf. (2.10)) but rather the symplectic combination of the relevant pairs of time- $t$ Cauchy data

$$
\sigma\left(\left(\varphi_{1}, \pi_{1}\right) ;\left(\varphi_{2}, \pi_{2}\right)\right)=\left\langle\varphi_{1} \mid \pi_{2}\right\rangle-\left\langle\pi_{1} \mid \varphi_{2}\right\rangle
$$

(here $\langle 1\rangle$ denotes the inner product in $\mathscr{H}(3.4))$. Thus it is reasonable to enquire whether (for a given mass $\mu$ ) in addition to the $\mathscr{T}_{\mu}^{(q)}(t)$ constructed above there may not be further "exotic" options for ( $z$-translationally-invariant) acceptable global time evolutions. In Sect. A2, we pose this question in a precise form, but do not answer it. (We shall ignore the possibility of such "exotic" options in the remainder of this paper.)

Here, we give a brief discussion of the global time evolutions $\mathscr{T}_{\mu}^{(q)}$ which we have constructed. The qualitative behaviour will depend crucially on whether or not $A_{\mu}^{(q)}(3.4)$ is a positive operator. In the positive case, $0 \leqq q \leqq \mu$, the conserved energy

$$
\mathscr{E}_{\mu}^{(q)}(\varphi, \pi)=\frac{1}{2}\left(\langle\pi \mid \pi\rangle+\left\langle\varphi \mid A_{\mu}^{(q)} \varphi\right\rangle\right)
$$

is positive, indicating good (i.e. bounded $\langle 18\rangle$ ) large-time behaviour. When $A_{\mu}^{(q)}$ fails to be positive (i.e. when $q>\mu$ ) on the other hand, one expects there to be initially small solutions (say with $C_{0}^{\infty}$ initial data) which grow exponentially at late times ("runaway solutions"). To demonstrate this, it is convenient to first consider the $z$-integral

$$
\tilde{\phi}(t, \varrho, \theta)=\int_{-\infty}^{\infty} \phi(t, \varrho, \theta, z) d z
$$

of such a solution, which will of course obey the $1+2$ dimensional Klein-Gordon equation

$$
\dot{\tilde{\phi}}+\left(\mu^{2}-\Delta^{(q)}\right) \tilde{\phi}=0
$$


( $\Delta^{(q)}$ as in Sect. 2.) Assuming that $q \tilde{\phi}+\dot{\widetilde{\phi}}$ and the bound state $\psi_{\text {bound }}^{(q)}(2.6)$, have at some initial time non-vanishing overlap $\left\langle q \tilde{\phi}+\dot{\phi} \mid \psi_{\text {bound }}^{(q)}\right\rangle$ (in the inner-product (2.10)) $\tilde{\phi}$ will split at late times into the sum of a well-behaved (we suppose bounded $\langle 18\rangle)$ piece and a non-zero runaway component

$$
\text { const } \exp \left(\left(q^{2}-\mu^{2}\right)^{1 / 2} t\right) \psi_{\text {bound }}^{(q)}
$$

which would first start to become noticeable around the time the wave packet first hit the string. Using finite propagation speed (in the $z$-direction) to estimate the integral (3.9), one infers that the solution itself will satisfy

$$
|\phi| \geqq \frac{\text { const }}{t} \exp \left(\left(q^{2}-\mu^{2}\right)^{1 / 2} t\right)\left|\psi_{\text {bound }}^{(q)}\right|
$$

Thus for the non-positive self-adjoint extensions of $A_{\mu}$ (3.2) the dynamics is expected to be classically unstable. (Note however, that, as we shall explain in Sect. 5, in modelling the behaviour of waves propagating on true strings, this instability in the idealized model can sometimes be spurious.)

\section{The Klein-Gordon Quantum Field Theory Around an Idealized String}

In this section, we discuss the mathematical options for quantizing (1.2), continuing to treat the case of a real scalar field. It is appropriate to distinguish between two levels of structure: the "algebraic structure" and the "ground state structure" (cf. e.g. the general discussion of quantum field theory in curved spacetime in [30] or the more recent discussion in Sect. 3 of [22]). We shall show that, for each choice of classical dynamics $\mathscr{T}_{\mu}^{(q)}(t)$ constructed in Sect. 3, will correspond a quantization of (1.2) at the algebraic level by taking the equal-time CCR algebra over $D_{\mu}^{(q)}(3.5)$ and imposing the classical time evolution $\mathscr{T}_{\mu}^{(q)}(t)$ on the quantum $\hat{\varphi}$ 's and $\hat{\pi}$ 's. However, not all these possible algebraic quantizations will admit a ground state. For this (as for classical stability) we shall see that positivity of the operator $A_{\mu}^{(q)}(3.3)$ will be needed.

To make precise the quantization at the algebraic level (cf. e.g. $[23,22]$ ) we begin by defining the time-zero CCR algebra $\mathbf{A}_{\mu}^{(q)}$ as a $*$-algebra with identity $I$ generated by Hermitian (i.e. satisfying $\left.\hat{R}(\varphi, \pi)^{*}=\hat{R}(\varphi, \pi)\right)$ objects

$$
\hat{R}(\varphi, \pi), \text { with } \quad(\varphi, \pi) \in D_{\mu}^{(q)} \quad(\text { see }(3.5))
$$

(to be interpreted as the "symplectically smeared combination" - cf. (3.7) -

$$
\hat{R}(\varphi, \pi)=\langle\hat{\varphi} \mid \pi\rangle-\langle\hat{\pi} \mid \varphi\rangle=\sigma((\hat{\varphi}, \hat{\pi}),(\varphi, \pi))
$$

also writeable as $\hat{\varphi}(\pi)-\hat{\pi}(\varphi))$, satisfying, for all $\left(\varphi_{1}, \pi_{1}\right),\left(\varphi_{2}, \pi_{2}\right) \in D_{\mu}^{(q)}$,

$$
\left[\hat{R}\left(\varphi_{1}, \pi_{1}\right), \hat{R}\left(\varphi_{2}, \pi_{2}\right)\right]=i\left(\left\langle\varphi_{1} \mid \pi_{2}\right\rangle-\left\langle\pi_{1} \mid \varphi_{2}\right\rangle\right) I .
$$

We then represent the time-evolution by algebra automorphisms $\beta_{\mu}^{(q)}(t): \mathbf{A}_{\mu}^{(q)} \rightarrow \mathbf{A}_{\mu}^{(q)}$, mapping the time-zero fields to time- $t$ fields according to $\beta_{\mu}^{(q)}(t): \hat{R}(\varphi, \pi) \mapsto \widehat{R}_{t}(\varphi, \pi)$, where

$$
\widehat{R}_{t}(\varphi, \pi)=\hat{R}\left(\mathscr{T}_{\mu}^{(q)}(-t)(\varphi, \pi)\right)
$$

(with the interpretation $\hat{R}_{t}(\varphi, \pi)=\hat{\varphi}_{t}(\pi)-\hat{\pi}_{t}(\varphi)$ ). For each mass $\mu$, we thus have a whole family $\left(\mathbf{A}_{\mu}^{(q)}, \beta_{\mu}^{(q)}(t)\right)$ of inequivalent possible quantizations of $(1.2)$ at the 
algebraic level (or “*-dynamical systems") corresponding to the different possible options for the classical time evolution.

The next question is whether the system $\left(\mathbf{A}_{\mu}^{(q)}, \beta_{\mu}^{(q)}(t)\right)$ admits a ground state; equivalently whether $\mathbf{A}_{\mu}^{(q)}$ admits a representation $\hat{\varrho}_{\mu}^{(q)}$ on a (complex) Hilbert space $\mathscr{F}$ with "vacuum vector" $\Omega$ such that $\beta_{\mu}^{(q)}(t)$ is implemented by a (strongly continuous) positive-energy unitary group $\exp \left(-i H_{\mu}^{(q)} t\right)$ in the sense that

$$
\hat{\varrho}_{\mu}^{(q)}\left(\beta_{\mu}^{(q)}(t) B\right)=\exp \left(i H_{\mu}^{(q)} t\right) \hat{\varrho}_{\mu}^{(q)}(B) \exp \left(-i H_{\mu}^{(q)} t\right) \quad \forall B \in \mathbf{A}_{\mu}^{(q)}
$$

with $H_{\mu}^{(q)} \Omega=0$. The answer is that, over $\left(\mathbf{A}_{\mu}^{(q)}, \beta_{\mu}^{(q)}(t)\right)$ such a ground state structure can exist only for those $\mu$ and $q$ for which $A_{\mu}^{(q)}$ is positive (i.e. only when $0 \leqq q \leqq \mu$ ). In fact, one can argue quite generally that (setting aside possible subtle pathologies) for linear Bose systems such as ours, positivity of the classical energy is a necessary condition for the existence of a quantum ground state. We give a heuristic argument for this ignoring domain questions. (For a related, but maybe overrestrictive, rigorous result, see the Corollary to Lemma 6.1 in [22].) The argument (which generalizes to any linear Bose system) is based on the "infinitesimal" versions of (3.6) and (4.4) from which we have (now dropping sub- and superscripts)

$$
[i H, \hat{\varrho}(\hat{R}(\varphi, \pi))]=\hat{\varrho}(\hat{R}(\mathbf{h}(\varphi, \pi)),
$$

where $\mathbf{h}$ is the classical generator of time evolution (cf. (3.1), (3.6)) $\mathscr{T}(t)$,

$$
\mathbf{h}(\varphi, \pi)=(-\pi, A \varphi) .
$$

Assuming the existence of a ground-state structure, one then calculates that the manifestly positive expression

$$
\begin{gathered}
\langle\Omega \mid \hat{\varrho}(\hat{R}(\varphi, \pi)) H \varrho(\hat{R}(\varphi, \pi)) \Omega\rangle=\frac{1}{2}\langle\Omega|[\hat{\varrho}(\hat{R}(\varphi, \pi)),[H, \varrho(\hat{R}(\varphi, \pi)]] \Omega\rangle \\
=(\text { by }(4.5) \text { and }(4.2)) \frac{1}{2} \sigma((\varphi, \pi), \mathbf{h}(\varphi, \pi)),
\end{gathered}
$$

which, by (4.6) and (3.7), is precisely the classical energy (3.8). Thus we only expect a ground state to exist when $A_{\mu}^{(q)}$ is positive. We remark that in Sect. 3, this was argued to be the condition (modulo the fine point mentioned in Note $\langle 18\rangle$ ) for the classical time evolution to be stable. (As for the classical stability result, note, however, that caution is required in interpreting the significance of this condition when one uses these idealized quantizations to model the options for ground states on "true" strings. See the discussion in Sect. 5.)

To actually construct such a ground-state structure (see [24] and also Sect. 3 in [22] for the full general theory) we deal first with the cases where the spectrum of $A_{\mu}^{(q)}$ is bounded below away from zero so that $A_{\mu}^{(q)}$ has a bounded inverse. This will hold when $q<\mu$. In these cases, the key step is to define the following map $\left(\mathscr{H}_{\mathbb{C}}\right.$ below denotes the complex Hilbert space $L_{\mathbb{C}}^{2}\left(\mathbb{R}^{+} \times \mathbb{S} \times \mathbb{R}\right.$, $\left.\left.\varrho d \varrho d \theta d z\right)\right)$ :

$$
\begin{aligned}
K_{\mu}^{(q)}: D_{\mu}^{(q)} & \rightarrow \mathscr{H}_{\mathbb{C}} \\
(\varphi, \pi) & \mapsto 2^{-1 / 2}\left(A_{\mu}^{(q)^{1 / 4}} \varphi+i A_{\mu}^{(q)^{-1 / 4}} \pi\right) .
\end{aligned}
$$

This will have the property of intertwining the classical time-evolution $\mathscr{T}_{\mu}^{(q)}(t)$ with the positive energy unitary group $U(t)=\exp \left(-i A_{\mu}^{(q)^{-1 / 2}} t\right)$ so that $\mathscr{H}_{\mathbb{C}}$ may be regarded as a suitable one-particle Hilbert space (the elements $\psi$ of which satisfy the "relativistic Schrödinger equation" (cf. (1.3))

$$
i \partial \psi / \partial t=A_{\mu}^{(q))^{1 / 2}} \psi \text {. }
$$


One may then build up a ground-state representation $\hat{\varrho}_{\mu}^{(q)}$, taking as Hilbert space $\mathscr{F}$ the Fock space over $\mathscr{H}_{\mathbb{C}}$ with its Fock vacuum vector $\Omega$ by defining in the usual way

$$
\hat{\varrho}_{\mu}^{(q)}(\hat{R}(\varphi, \pi))=-i\left(a^{\dagger}\left(K_{\mu}^{(q)}(\varphi, \pi)\right)-\left(a^{\dagger}\left(K_{\mu}^{(q)}(\varphi, \pi)\right)^{*}\right),\right.
$$

where $a^{\dagger}$ is the usual creation operator on $\mathscr{F}$ satisfying

$$
\left[\left(a^{\dagger}\left(\psi_{1}\right)^{*}\right), a^{\dagger}\left(\psi_{2}\right)\right]=\left\langle\psi_{1} \mid \psi_{2}\right\rangle, \quad\left(a^{\dagger}(\psi)\right)^{*} \Omega=0 .
$$

Thus, for $\mu>0$, corresponding to each value of $q$ in the range $0 \leqq q<\mu$ there will be a distinct possible quantum dynamics admitting a ground state $\langle 5\rangle$. Different such choices of $q$ will, of course, correspond to different "physics." This is clearly seen if one calculates the spatially smeared two-point function

$$
\begin{aligned}
" \iint\langle & \left\langle\Omega \mid \hat{\varrho}_{\mu}^{(q)}\left(\hat{\varphi}\left(\varrho_{1}, \theta_{1}, z_{1}, t\right) \hat{\varphi}\left(\varrho_{2}, \theta_{2}, z_{2}, 0\right)\right) \Omega\right\rangle f_{1}\left(\varrho_{1}, \theta_{1}, z_{1}\right) f_{2}\left(\varrho_{2}, \theta_{2}, z_{2}\right) \\
& \times \varrho_{1} d \varrho_{1} d \theta_{1} d z_{1} \varrho_{2} d \varrho_{2} d \theta_{2} d z_{2} " \\
= & \left\langle\Omega \mid \hat{\varrho}_{\mu}^{(q)}\left(\hat{R}_{t}\left(0, f_{1}\right) \hat{R}_{t}\left(0, f_{2}\right)\right) \Omega\right\rangle_{\mathscr{F}} \\
= & \left\langle f_{1}\right|\left(\exp \left(-i A_{\mu}^{(q))^{1 / 2}} t\right)\left(2 A_{\mu}^{(q)}\right)^{-1 / 2} f_{2}\right\rangle_{\mathscr{H}_{\mathbb{C}}},
\end{aligned}
$$

which essentially characterizes the theory. In particular, one may use (4.9) as a starting point for a computation of the renormalized ground state expectation value of the energy-momentum tensor operator (cf. [25] where the case $\mu=0, q=0$ is treated in detail. Note that [25] also calculates an explicit analytic form for (4.9) in this case confirming the result of [6].) One expects of course to obtain different results for different values of $q$. (See also the remarks at the end of Sect. 5.2.)

So far, we have left out of consideration the "borderline cases" $q=\mu$, where the spectrum of $A_{\mu}^{(q)}$ begins at zero. As with the discussion of classical stability (see Note $\langle 18\rangle$ ) one expects the technical issues here to be more delicate. In the important special case of zero mass and zero $q$ (the Friedrichs extension of (3.2)) the existence of a ground-state structure may be established by replacing the domain $D_{\mu}^{(q)}(3.5)$ in the case $\mu=0, q=0$ by

$$
D_{0}^{(0)}=\mathscr{D}\left(\left|A_{0}^{(0)}\right|^{1 / 2}\right) \oplus \mathscr{D}\left(\left|A_{0}^{(0)}\right|^{-1 / 2}\right) .
$$

One easily checks that this is an invariant domain for $\mathscr{T}_{0}^{(0)}(t)(3.6)$. Also the map $K_{0}^{(0)}(4.7)$ and the formula for the two-point function (4.9) will make sense on this domain. Finally, one checks that $D_{0}^{(0)}$ is an extension of the domain of smooth, compactly supported, time- $t$ Cauchy data $C_{0}^{\infty}\left(\mathbb{R}^{+} \times \mathbf{S} \times \mathbb{R}\right) \times C_{0}^{\infty}\left(\mathbb{R}^{+} \times \mathbf{S} \times \mathbb{R}\right)$. The non-trivial property that has to be checked for this latter to hold is

$$
C_{0}^{\infty}\left(\mathbb{R}^{+} \times \mathbf{S} \times \mathbb{R}\right) C \mathscr{D}\left(\left|A_{0}^{(0)}\right|^{-1 / 2}\right) .
$$

We give a proof that this holds for cones with $0<\kappa \leqq 1$ in Note $\langle 19\rangle$.

We conclude that there is a unique (up to the issues raised in Note $\langle 5\rangle$ ) $z$-translationally-invariant quantization admitting a ground state in the case of zero mass. One easily sees that this quantization corresponds to that of Ref. [4-10,38]. Note however, that, in Sect. 5, we shall point out that, if one is interested in modelling the "true" quantum ground state on certain types of "true" cosmic string, the restrictions on possible $q$-values obtained above for our idealized string (and in particular the implication that there is a unique possibility in the case of zero mass) may be misleading. We shall briefly discuss there how one could e.g. better plausibly model the ground state for a massless field on such a true string with a non-zero $q$-value. 


\section{Quantum Mechanics on Rounded-off Cones and Fields Around "True" Strings}

In this section, we discuss the relevance of the various idealized problems treated in Sects. 2-4 to problems such as the Schrödinger time-evolution of wavefunctions on a rounded-off cone and (classical and quantum) field theory around a "true" cosmic string of finite thickness (still assumed to be infinitely long, straight and static). Such a true string will of course itself consist of a special configuration of fields interacting with each other according to some suitable theory. To a good approximation, this configuration will be describable as classical and smooth. We shall restrict our attention to models for strings where it is also a good approximation to assume that these fields are confined to a narrow tubular region (and, we shall assume, translationally invariant in $z$ ). Typically these are "local strings." The gravitational field of such a string will also be essentially confined to the same narrow tubular region where the metric will be curved but smooth - the spacetime outside the tubular region becoming rapidly isometric to the locally flat spacetime $(\mathscr{M}, g)(1.1)$. (Appropriate references for the above remarks are e.g. $[1,3$, $35-38,48,54,55]$.) To get closer to true physics, one should replace the model discussed in Sects. 3 and 4 by a (we shall assume still scalar and linear) field $\phi$ propagating on the spacetime of such a true string and interacting either only with the gravitational field or also with the other "matter" fields making up the string. Motivated by these remarks (but simplifying the model a little further) we shall intend in what follows by the "interaction of a true string with our scalar field $\phi$ " the equation

$$
\left(\square_{\tilde{g}}+\tilde{V}+\mu^{2}\right) \phi=0 .
$$

Here $\square_{\tilde{g}}$ is the Laplace-Beltrami operator for the spacetime $\left(\tilde{\mathscr{M}} \approx \mathbb{R}^{4}, \tilde{g}\right)$, where

$$
\tilde{\mathrm{g}}=d t^{2}-\tilde{h}-d z^{2}
$$

with $\tilde{h}$ the metric of a "rounded-off cone" - i.e. some circularly symmetric smooth metric on $\mathbb{R}^{2}$, which with a suitable choice of polar coordinates $(\varrho, \theta)$ coincides outside a certain value of $\varrho$ (we shall say outside its "roundedness support") precisely with $h(1.5)$ and $\widetilde{V}$ is a smooth (we shall assume circularly symmetric and translationally invariant) "potential" with compact support which represents a possible interaction with the "matter" fields in the string or (through $\widetilde{V}=$ const $R$, where $R$ is the Riemann scalar for $\widetilde{h}$ ) a non-minimal coupling to the gravitational field. Explicitly, $\tilde{h}$ will be given by

$$
\tilde{h}=d \varrho^{2}+\kappa^{2}(\varrho) \varrho^{2} d \theta^{2},
$$

where $\kappa(\varrho)$ is a smooth function on $\mathbb{R}^{+}$(smoothly extendable to a smooth symmetric function on $\mathbb{R}$ ) which takes the value 1 at $\varrho=0$ and the constant value $\kappa$ outside the roundedness support.

We shall thus be interested in the relationship between the discussion of Eq. (1.2) in Sects. 3 and 4 and the dynamics of (5.1). Similarly, we shall attempt to relate the discussion of Eqs. (1.4) and (1.5) in Sect. 2 with the $1+2$ dimensional equations

$$
i \frac{\partial \psi}{\partial t}=\left(-\Delta_{\tilde{h}}+\tilde{V}\right) \psi
$$


and

$$
i \frac{\partial \psi}{\partial t}=\left(\mu^{2}-\Delta_{\tilde{h}}+\tilde{V}\right)^{1 / 2} \psi
$$

Note that in (5.5) some condition is required on $\widetilde{V}$ to ensure the positive squareroot exists. A sufficient condition would be that $\widetilde{V}$ were bounded below by $-\mu^{2}$. In parallel to the "idealized problems A, B, C, D of the introduction, we shall thus have the counterpart "true" problems $-\widetilde{\mathrm{A}}, \widetilde{\mathrm{B}}, \widetilde{\mathrm{C}}, \widetilde{\mathrm{D}}$ say. For these latter true problems, there is of course no need to impose boundary conditions at $\varrho=0$; each of Eqs. (5.1), (5.4), and (5.5) determines a unique dynamics - with suitable smooth initial data remaining smooth under time-evolution.

As we discussed in the introduction, it seems natural to make the hypothesis that, for each true problem $(\widetilde{\mathrm{A}}, \widetilde{\mathrm{B}}, \widetilde{\mathrm{C}}$, or $\widetilde{\mathrm{D}})$ - and each specific choice of $\tilde{h}$ and $\widetilde{V}$ there will be a particular allowed $R$-value (equivalently $q$-value, see Note $\langle 4\rangle$ ) for the corresponding idealized problem $(\mathrm{A}, \mathrm{B}, \mathrm{C}$, or $\mathrm{D})$ for which the idealized dynamics well approximates the true dynamics at sufficiently large length scales. In the remainder of this section, we shall discuss the extent to which, and sense in which, this hypothesis is true. Section 5.1 contains the main ideas and our principal result. In Sect. 5.2 we find that, when one looks how things work out in detail, a number of subtle points arise. We shall attempt to clarify these (see also Note $\langle 22\rangle$ to Sect. 5.1) paying special attention to the case of large positive potentials, which is relevant to the scattering of electromagnetic waves on superconducting cosmic strings. Further discussion of closely related issues in a more general context will appear in [42] (see also [43]).

5.1. Main Ideas and Principal Result. We turn first to non-relativistic quantum mechanics on a "true" cone (Eq. (5.4)). For this problem, the sense in which our hypothesis holds may be summarized by the following two points:

1. For a given choice of metric $\tilde{h}$ and potential $\tilde{V}$ in (5.4) one should choose the $R$-value for quantum mechanics on an idealized cone (1.4) so as to match the logarithmic derivatives (i.e. $\psi^{-1} \partial \psi / \partial \varrho$ ) of the static (i.e. "zero-energy") circularlysymmetric (non-normalizable) solutions to the true and idealized problems at any radius $\varrho=a$ which is outside both the roundedness support of $\tilde{h}$ and the support of $\widetilde{V}$.

2. The idealized dynamics for the resulting $R$-value will then well-approximate the true dynamics at sufficiently large length scales.

In clarification of point (1), we first remark that, for the true problem, we assume our static circularly-symmetric solution, $\psi_{\text {true }}^{\text {stac }}$ say, to (5.4) to be smooth. It will then be unique up to a constant, since it will be determined by integrating the radial equation

$$
\left(-\frac{1}{\kappa(\varrho) \varrho} \frac{\partial}{\partial \varrho} \kappa(\varrho) \varrho \frac{\partial}{\partial \varrho}+\widetilde{V}(\varrho)\right) \psi_{\text {true }}^{\text {static }}=0
$$

using the property (as opposed to "boundary condition"!) that $\psi_{\text {true }}^{\text {static }}$ will be finite at $\varrho=0$. On the other hand, for the idealized dynamics labelled by $R$, i.e. with the boundary condition (2.3)/(1.8), one will obtain (by (1.7) and (2.3)) the solution, $\psi_{R}$ 
say,

$$
\psi_{R}=\text { const } \ln (\varrho / R) \text {. }
$$

(In the special case $R=0,(5.7)$ should be replaced by $\psi_{0}=$ const.)

Outside the radius $\varrho=a, \psi_{\text {true }}^{\text {static }}$ will, of course, take the form of Eq. (5.7) for some $R$. This is the $R$-value which our procedure selects. It is clearly determined by the "fitting formula"

$$
R=a \exp \left(-\frac{1}{a} \frac{\psi_{\text {true }}^{\text {static }}}{\partial \psi_{\text {true }}^{\text {static }} / \partial \varrho} \uparrow_{\varrho=a}\right)
$$

For a given $\tilde{h}$ and $\widetilde{V}$, we shall refer to the parameter $R$, obtained in this way, as the "scattering length," since it is closely analogous to the familiar three-dimensional notion $\langle 20\rangle$.

Concerning point (2), we remark that the procedure in (1) may be viewed as fitting the dynamics $\langle 21\rangle$ of the idealized problem to coincide with that of the true problem for scattering states at zero momentum. In consequence, one expects it will continue to approximate the true dynamics well at sufficiently low momenta or energies. This is what we mean by "sufficiently large length scales." On the other hand, for a given true problem, one would not expect features of the fitted idealized dynamics which have to do with small scales to be believable. It is of course of great importance, in applying this idea to practical calculations to make this notion more quantitative by expressing the believability of the fit at any given scale as a function of the parameters of the potential. Such matters will be discussed further in [42].

Our principal result is that, in the case $\tilde{V}=0$, the above fitting procedure gives an $R$-value of zero. The proof is extremely simple: Using (5.6) one sees that a static circularly symmetric solution of (5.4) with $\widetilde{V}=0$ will satisfy

$$
\frac{\partial}{\partial \varrho} \kappa(\varrho) \varrho \frac{\partial \psi_{\text {true }}^{\text {static }}}{\partial \varrho}=0 \text {. }
$$

This is uniquely solved (again using the property of finiteness of at $\varrho=0$ ) by $\psi_{\text {true }}^{\text {static }}$ = constant.

Thus it is correct (in the sense that one will obtain a good approximation at sufficiently large length-scales) to model (minimally coupled) quantum mechanics on a rounded-off cone with no potential term by the "usual" self-adjoint extension $-\Delta^{0}$ of $-\Delta$ on an idealized cone $\langle 22\rangle$.

Turning to the true classical Klein-Gordon equation (our true problem $\widetilde{\mathbf{B}}$ ) one expects the true dynamics for Eq. (5.1) to be well approximated by the idealized dynamics described in Sect. 3 where one simply chooses the parameter $R$ to fit the potential $\widetilde{V}$ in the corresponding $1+2$ dimensional non-relativistic problem. In particular, our above principal result implies that the value $R=0$ will be equally appropriate for approximating the dynamics of Eq. (5.1) in the case $\widetilde{V}=0$. Similar remarks apply to our true problems $\widetilde{\mathrm{C}}$ and $\tilde{\mathrm{D}}$.

5.2. Non-Zero R-values and Superconducting Cosmic Strings. We now look in a little more detail at the case where there is a non-zero $\widetilde{V}$ term in Eq. (5.1) or (5.4), in which cases, according to the above discussion, one expects the idealized dynamics for non-zero values of the $R$-parameter to be relevant. Some of the points we make below will be very brief. For further discussion see [42]. 
We begin with a number of (easily demonstrated) observations concerning the question of which $R$-values fit which $\widetilde{V}$. We continue to confine the discussion to spherical symmetry and assume $\widetilde{V}$ is supported inside some radius $a$.

1. In the case that $\tilde{V}$ is non-negative, it follows from the fitting formula (5.9) that $R$ is bounded by $a$.

2. A true potential $\widetilde{V}$ which is non-positive will always have at least one bound state. By varying the depth of such a potential, one may achieve any value of $R$. As the depth crosses a threshold for a new bound state, the fitted $R$-value jumps from zero to infinity. (Since such potentials always bind, the first such threshold is at zero potential!)

3. For sufficiently weak potentials (the criterion is that the integral in (5.10) below should be very much less than one) the fitted $R$ will be well approximated by

$$
R \approx a \exp \left(-\kappa / \int_{0}^{a} \tilde{V}(\varrho) \kappa(\varrho) \varrho d \varrho\right) .
$$

In the case of a weak non-negative potential, we remark that the formula (5.10) leads to a value of $R$ which is "exponentially smaller" than $a$. As a tool to approximate the physics at large scales (i.e. at scales much larger than $a$ ) the idealized dynamics for such a value of $R$ will differ little from that for $R=0$, and, for many purposes, it would be reasonable simply to take $R=0$.

In the case of a very weak non-positive potential, it may at first sight appear paradoxical that one obtains, from (5.10), values of $R$ which are exponentially large. The resolution is that at "medium scales" (i.e. a few orders of magnitude larger than $a$ but not exponentially larger than $a$ ) the dynamics for such a value of $R$ will differ very little from the dynamics for the case $R=0$, and again it would often be reasonable to simply approximate the true dynamics - as far as such medium scales are concerned - by the idealized dynamics for $R=0$. This is related to the fact (see Note $\langle 4\rangle$ and the remarks at the end of Appendix A) that, with a suitable notion of limit, the limit as $R$ tends to infinity of the idealized dynamics labelled by $R$ is equal to the idealized dynamics for $R=0$. (For a fuller understanding of this point, see the discussion of "effect" versus scale for twodimensional potentials in [42].)

We remark that, in the relativistic case, Eq. (5.1), with any true potential $\tilde{V}$ for which the operator $-\Delta_{\tilde{h}}+\widetilde{V}$ has a bound state, one easily sees by a similar argument to that given for the idealized dynamics in Sect. 3, that, in the case, say of a massless field $\phi$, there will be runaway solutions. Thus one would expect massless fields on true cosmic strings not to be modelled by such potentials (and in particular not to be modelled by non-zero non-positive potentials) since if they were, they would be expected to lead to an instability of the string (although for sufficiently weak such potentials, the characteristic time could be very large). Note in fact that, in the case of a very weak non-positive potential, a good approximation for $q$ will (by (1.7) and (5.10)) be

$$
q \approx 2 e^{-c} a^{-1} \exp \left(\kappa / \int_{0}^{a} \tilde{V}(\varrho) \kappa(\varrho) \varrho d \varrho\right),
$$

so that the bound state energy will be (see after Eq. (2.6)) $-q^{2}$ and the characteristic time for the runaway solution for the massless relativistic field will be (by (3.11)) $q^{-1}$ for this $q$-value. 
It may at first sight appear paradoxical that, even in the case of true potentials which are non-negative (and hence have no bound states) the fitted idealized dynamics will (since $R$ will never be precisely zero) always have a bound state. The resolution is that, in view of the bound $R \leqq a$, the bound state (with energy, by (1.7), $-q^{2}=-4 e^{-2 c} R^{-2}$ ) is a feature of the idealized dynamics which has to do with length scales of the order of $a$ where, as we discussed above, the fit should not be expected to be believable. Thus, for example, when one uses the idealized dynamics for a non-zero $R$-value to approximate the behaviour of (say massless) waves in interaction with a cosmic string described by a non-negative $\widetilde{V}$, the runaway solutions derived in Sect. 3 should be regarded as spurious. We shall return to this point at the end of this section.

We now focus attention on the case of potentials $\widetilde{V}$ which are both non-negative and strong in the sense that the value of the integral in (5.10) is of the order of unity. For such potentials, the approximation (5.10) will break down, but one sees directly from the fitting formula (5.9) that the value of $R$ will now be of the order of (and no larger than) $a$ (rather than exponentially smaller than $a$ ). Such potentials are particularly interesting since, in the zero-mass relativistic case, they may be regarded as models for the dynamics of the electromagnetic field around a superconducting cosmic string. In fact, ignoring gravity (i.e. setting $\kappa=1$ - which we shall do from now on) and in the Lorentz gauge, each component of the vector potential $A^{v}$ will satisfy Eq. (5.1) in the case $\mu=0$ (see e.g. Eq. (43) in [52] $\langle 23\rangle$ ) where $\widetilde{V}$ now represents the "local mass" term for the photon inside the string.

Possible mechanisms for such a term are e.g. cosmic strings with certain types of non-abelian gauge-field configurations [57] or with an electrically charged Higgs field with a non-vanishing vacuum expectation value inside the string radius (the bosonic model in [52]). For other possible mechanisms see e.g. [52, 56, 57].

The point we wish to emphasize is that, to obtain a good approximation for the dynamics for such a potential at medium scales (i.e. a few orders of magnitude greater than $a$ ), it is important to take the idealized dynamics for the correct value of $R$, and not to replace $R$ by zero. The situation here is very different from the more familiar behaviour of (say spherically symmetric) positive potentials of small support in three dimensions, where again one sees (this time from the three dimensional fitting formula - see Note $\langle 20\rangle$ ) that the scattering length (this time $L$ as defined in Note $\langle 20\rangle$ ) is bounded by the radius of the support $a$. In this threedimensional case, it would make little difference at large scales whether one approximated the dynamics for such a potential by carefully fitting $L$ or simply by setting $L=0$. The reason is that, in three dimensions, one can show [42] that the "effect" of such a potential falls off at large scales as the inverse square of the scale. In two dimensions, on the other hand, the "effect" falls off only as the inverse square of the logarithm of the scale, and would thus still be considerable at scales a few orders of magnitude greater than $a$. (In these relations, the appropriate unit of scale is the scattering length.)

As an application and illustration of these ideas, note that, for a superconducting string with local mass term $\widetilde{V}$ one expects a good approximation to the scattering cross section per unit length of string for $z$-translationally-invariant electromagnetic waves to be given by (2.16),

$$
\left.2 \pi\left|f_{p}\right|^{2}=\pi^{2} p^{-1}(\ln [q / p])^{2}+\pi^{2} / 4\right)^{-1},
$$

where $q$ is determined by Eq. (1.7) and the fitting formula (5.9). (Comparison with Eq. (55) in [52] shows that the parameter $\Lambda$ in [52] should be identified with this value of $q$.) 
Finally, we point out that the special features of two-dimensional potentials that we have discussed above (see also "Pitfalls 2 and 3" in Note $\langle 22\rangle$ ) imply that sometimes (notably in the case of the problems we denoted by D and D) the relation between the idealized and "true" systems is not as clearcut as would be suggested by our initial "natural hypothesis." (This was the hypothesis that, for each "true" problem, there will be a particular allowed $R$-value for the corresponding idealized problem which led to a good approximation.) Consider the situation for quantizations admitting ground states of Eq. (5.1) - again in the case where $\mu=0$ and $\widetilde{V}$ is a strong non-negative potential. One knows that each such true problem will have a quantization admitting a ground state. (We shall assume our quantizations to have vanishing one-point functions. Up to possible fine points which we wish to de-emphasize here, these will then be unique, cf. Note $\langle 5\rangle$.) For the corresponding idealized system $\left(\square_{g} \phi=0\right.$ on an idealized cone) we showed, in Sect. 4 , that there is a unique allowed $R$-value (namely $R=0$ ) for which this admits a ground state. If one would take our hypothesis literally for this case, it would seem to suggest that the vacuum states for all strings in the class we are considering of a given radius $a$ (i.e. independently of the values of $\tilde{h}$ and $\widetilde{V}$ inside the string radius) should coincide to a good approximation at large scales. The fact is, however, that (again because of the logarithm in the relation between "effect" and length scale) one expects the approximation will only be good at exponentially large scales, and not "good enough" at "medium scales."

To understand why our hypothesis fails to apply very well in this case, one must recall the reason why $R=0$ is the only allowed $R$-value for the idealized problem: This may clearly (see Sect. 4) be traced back to the fact that, for all other $R$-values, $-\Delta^{R}$ fails - because of its bound state - to have a positive spectrum. However, the bound state of $-\Delta^{R}$ will (when one is modelling a non-negative potential of small support) have to do with very small length and time scales where the idealized dynamics is not a believable approximation to the true dynamics. The failure of our idealized system for the appropriate fitted $R$-value to admit a ground state should thus be regarded as spurious, in the same way that, as argued above, the classical runaway solutions in the fitted idealized dynamics to the corresponding classical problem should be regarded as spurious.

In order to overcome this problem and obtain a better approximation to the vacuum state of (5.1) at medium length scales, one reasonable way to proceed would perhaps be to introduce a modified idealized dynamics in which one simply projects out the bound state, i.e. replaces $-\Delta^{R}$-for the appropriate fitted $R$-value - by $-\Delta^{R}\left(I-P_{b}\right)$, where $P_{b}$ is the one-dimensional projector onto the bound state. (This new dynamics would be non-local, but the non-locality would be only on "small" length scales of the order of the string radius $a$.) If one were to follow through the construction of Sect. 4 , replacing $-\Delta^{R}$ by $-\Delta^{R}\left(I-P_{b}\right)$, one would presumably obtain a "modified" vacuum state which much more accurately approximates the true vacuum state at medium scales. (The bound state - which would now be a zero mode - would require special treatment. There would also be some additional infra-red technicalities to investigate - cf. Note $\langle 19\rangle$.) It may then be interesting e.g. to calculate the expectation value of the energy-momentum tensor in the vacuum states which one obtains in such a way for non-zero $R$-values. Note however that the question of "believability" for quantities such as the energymomentum tensor is expected to be particularly problematic, since it involves an integral over all scales, and it may be that, for certain types of interaction, one cannot satisfactorily summarize the effect of the $\widetilde{V}$ term by a single parameter. In 
order to investigate this question, it would be interesting to study further the recent results obtained by Allen and Ottewill [58] who calculate the renormalized expectation value of $\phi^{2}$ in the case of a non-minimally coupled (massless) scalar field on various types of model cosmic strings with rounded-off interior metrics. In particular, it would be interesting to see how well their results could be "mocked up" by choosing the appropriate non-zero $R$-value for an idealized string, as discussed above.

\section{Appendices}

\section{A1. Details on the Self-Adjoint Extensions of the Cone Laplacian}

We give some mathematical details behind the results in Sect. 2 on the self-adjoint extensions of $-\Delta(1.6)$ on the domain

$$
D=C_{0}^{\infty}\left(\mathbb{R}^{+} \times \mathbb{S}\right) C \mathscr{H}=L^{2}\left(\mathbb{R}^{+} \times \mathbb{S}, \varrho d \varrho d \theta\right) .
$$

Especially, we justify in detail all the statements up to Eq. (2.3). We shall rely on some results in [17] and [18] and especially in the Appendix to Sect. X.1 in [18] which we shall denote below by $[X]$. In the special case of deficit angle zero $(\kappa=1)$ an alternative discussion (leading to equivalent and further results) may be found in Chap. I.5 of [28], cf. also the discussion of related topics in [19].

A1.1. First, we prove that the self-adjoint extensions of this operator are the same as those of $-\Delta$ on the somewhat smaller "product domain" (cf. [17] Sect. VIII.10)

$$
\tilde{D}=C_{0}^{\infty}\left(\mathbb{R}^{+}\right) \otimes D_{\mathbf{S}}
$$

consisting of finite linear combinations of functions of the form $f(\varrho) g(\theta)$, where $f \in C_{0}^{\infty}\left(\mathbb{R}^{+}\right), g \in D_{\mathbf{S}}$,

$$
\text { where } D_{\mathbf{S}}=\left\{\sum_{m} a_{m} \exp (i m \theta): m \in \mathbb{Z} \text {, finitely many } a_{m} \neq 0\right\} .
$$

To show this, it clearly suffices to show that both operators have the same closure, i.e. that for all $\psi \in D$, one can find a sequence $\left\{\psi_{i}\right\} \in \widetilde{D}$ such that

$$
\psi_{i} \rightarrow \psi \text { and }-\Delta \psi_{i} \rightarrow-\Delta \psi \text { in } \mathscr{H} \text {. }
$$

We use a geometrical trick to reduce the proof of this to a standard theorem (Theorem VIII.33 in [17]) which says that a polynomial in essentially self-adjoint operators is essentially self-adjoint on the appropriate product domain. The trick is inspired by the observations: (i) that the lack of essential self-adjointness of $-\Delta$ is "caused by" the failure of our Riemannian manifold $(\mathscr{C}, h)(1.5)$ to be complete - a failure which is due to its behaviour near the (missing!) point $\varrho=0$. (Note that it is the missing point which causes the problem; the problem persists for $\kappa=1$ when there is no conical singularity.) (ii) That the support of any $\psi$ in $C_{0}^{\infty}\left(\mathbb{R}^{+} \times \mathbf{S}\right)$ will have a minimum radius $\varrho_{\min }$, and the existence of the sequence $\left\{\psi_{i}\right\}$ will be unaffected if we modify the geometry of the cone at smaller radii. The trick is as follows: First choose $\psi$ (with its $\varrho_{\min }$ ) and choose $\varrho_{0}$ with $0<\varrho_{0}<\varrho_{\min }$. Then consider minus the Laplace-Beltrami operator, $-\Delta^{\prime}$ on the (now complete) Riemannian manifold consisting of $\mathbb{R} \times \mathbb{S}$ with metric

$$
d s^{2}=d \varrho^{2}+r(\varrho)^{2} d \theta^{2},
$$


where $r(\varrho)$ is smooth, everywhere positive, and equal to $\varrho$ for $\varrho \geqq \varrho_{0}$ while it takes a constant positive value for all negative $\varrho .-\Delta^{\prime}$ on the domain $\widetilde{D}^{\prime}=C_{0}^{\infty}(\mathbb{R}) \otimes D_{\mathrm{S}}$ in $\mathscr{H}=L^{2}(\mathbb{R}, r d \varrho d \theta)$ consists of the polynomial

$A \otimes \mathbb{I}+B \otimes C \quad$ where $\quad A=-\frac{\partial^{2}}{\partial \varrho^{2}}-\frac{1}{r} \frac{d r}{d \varrho} \frac{\partial}{\partial \varrho}, \quad B=\frac{1}{r^{2}}, \quad$ and $\quad C=-\frac{\partial^{2}}{\partial \theta^{2}}$.

One easily shows that $A$ and $B$ are essentially self-adjoint on $C_{0}^{\infty}(\mathbb{R}) \subset L^{2}(\mathbb{R}, r d \varrho)$ (for $A$, one could use the methods in [26] as used in [24]) and that $C$ is essentially self-adjoint on $D_{\mathbf{S}}$. Thus, extending $\psi$ from $\mathbb{R}^{+}$to $\mathbb{R}$ by setting it to zero for negative $\varrho$ and applying Theorem VIII.33 in [17], (A1.1) must hold with $\widetilde{D}$ replaced by $\widetilde{D}^{\prime}, \mathscr{H}$ by $\mathscr{H}^{\prime}$ and $-\Delta$ by $-\Delta^{\prime}$. Clearly the resulting sequence $\left\{\psi_{i}\right\}$ can be chosen so as to have support in $\varrho>\varrho_{0}$ and will thus satisfy (A1.1) in its original version.

A1.2 (cf. Example 4 in [X]). Defining $L_{m}=\mathscr{L} \otimes K_{m}$, where $\mathscr{L}=L^{2}\left(\mathbb{R}^{+}, \varrho d \varrho\right)$ and $K_{m}$ is the one-dimensional subspace of $L^{2}(\mathbf{S})$ spanned by $e^{i m \theta}$, we may clearly write $-\Delta$ on $\widetilde{D}$ in $\mathscr{H}$ as

$$
\bigoplus_{m \in \mathbb{Z}}-\Delta_{m} \otimes \mathbb{I} \quad \text { on } \quad \bigoplus_{m \in \mathbb{Z}}\left(C_{0}^{\infty}\left(\mathbb{R}^{+}\right) \otimes K_{m}\right) \subset \underset{m \in \mathbb{Z}}{\bigoplus_{m}} L_{m},
$$

where $-\Delta_{m}$ is given by (2.2). We now study each of the terms in this direct sum, or rather the equivalent operators

$$
-\Delta_{m} \quad \text { on } \quad C_{0}^{\infty}\left(\mathbb{R}^{+}\right) C \mathscr{L}=L^{2}\left(\mathbb{R}^{+}, \varrho d \varrho\right) .
$$

It will be useful to study the equivalent (under $U: L^{2}\left(\mathbb{R}^{+}, \varrho d \varrho\right) \rightarrow L^{2}\left(\mathbb{R}^{+}, d \varrho\right)$, $\varphi \mapsto \varrho^{1 / 2} \psi$ ) operator

$$
-\tilde{\Delta}_{m}=-\frac{d^{2}}{d \varrho^{2}}+\left(-\frac{1}{4}+\frac{m^{2}}{\kappa^{2}}\right) \frac{1}{\varrho^{2}}
$$

on $C_{0}^{\infty}\left(\mathbb{R}^{+}\right)\left(\tilde{\mathscr{L}}=L^{2}\left(\mathbb{R}^{+}, d \varrho\right)\right.$. By Theorem X.8 in [18] this is in the limit point case at $\infty$ for all $m \in \mathbb{Z}$. By Theorem X.10 in [18] it is also in the limit case at 0 for $m \neq 0$ $\langle 8\rangle$. However, for $m=0$, it is in the limit circle case at 0 since $-\Delta_{0}$ then takes the form $-d^{2} / d \varrho^{2}+V(\varrho)$, where $V$ decreases with decreasing $\varrho$ (see Problem 7 in [18]). We conclude that, for $m \neq 0,-\Delta_{m}$ is essentially self-adjoint, whereas for $m=0$, it has deficiency indices $\langle 1,1\rangle$ and hence a one-parameter family of self-adjoint extensions. In consequence, cf. Problem 1a in Sect. X of [18] - the original operators $-\Delta$ on $\widetilde{D}$, and by Sect. A1.1, $-\Delta$ on $D$ will also have deficiency indices $\langle 1,1\rangle$, and hence also have a one-parameter family of self-adjoint extensions.

A1.3. To explicitly characterize all these self-adjoint extensions of $-\Delta_{0}$, we first note that one easily shows (e.g. applying Proposition 2 in $[\mathrm{X}]$ to $\tilde{\Delta}_{0}$ ) that the domain $\mathscr{D}\left(-\Delta_{0}^{*}\right)$ of the adjoint (which certainly extends all the self-adjoint extensions) consists of functions $\psi$ in $\mathscr{L}$ which satisfy

(i) $\psi$ is continuously differentiable, $\psi^{\prime}\left({ }^{\prime}=d / d \varrho\right)$ is almost everywhere differentiable and $-\Delta_{0}^{*}$ acts "classically" - i.e. as

$$
-\Delta_{0}^{*} \psi=-\psi^{\prime \prime}-\frac{1}{\varrho} \psi^{\prime}
$$


(ii) Moreover, by the calculations,

$$
\int_{0}^{a} \varrho^{\varepsilon}|\psi| d \varrho=\left\langle\varrho^{-1+\varepsilon} \chi_{[0, a)} \| \psi \mid\right\rangle_{\mathscr{L}}<\infty
$$

and

$$
\int_{0}^{a}\left|\left(\varrho \psi^{\prime}\right)^{\prime}\right| d \varrho=\int_{0}^{a}\left|\varrho\left(\psi^{\prime \prime}+\varrho^{-1} \psi^{\prime}\right)\right| d \varrho \leqq\left\langle\chi_{[0, a]} \| \Delta_{0}^{*} \psi \mid\right\rangle_{\mathscr{L}}<\infty,
$$

we have that both $\varrho^{\varepsilon} \psi$ for all $\varepsilon>0$, and $\varrho \psi^{\prime}$ are bounded in the vicinity of $\varrho=0$.

Using Property (i), one finds that the two normalizable functions (with equal norms)

$$
\psi_{ \pm}(\varrho)=K_{0}\left(2^{-1 / 2}(1 \mp i) \varrho\right)
$$

$\left(K_{0}\right.$ denotes the appropriate Bessel function of the third kind of imaginary argument [32]) satisfy

$$
-\Delta_{0}^{*} \psi_{ \pm}= \pm i \psi_{ \pm}
$$

and hence span the deficiency subspaces of $-\overline{\Delta_{0}}$ (here, the bar denotes closure). Standard theory (we use a trivial extension of Corollary (6) to Theorem X.2 in [18]) now assures us that the self-adjoint extensions (which we temporarily denote $\left.\left\{-\Delta_{0}^{[\delta]}: \delta \in \mathbb{C},|\delta|=1\right\}\right)$ of $-\Delta_{0}$ stand in one-to-one correspondence with complex numbers $\delta$ of modulus 1 - a core for the extension $-\Delta_{0}^{[\delta]}$ being

$$
D_{\delta}=\left\{\psi+c\left(\psi_{+}+\delta \psi_{-}\right): \psi \in C_{0}^{\infty}\left(\mathbb{R}^{+}\right), c \in \mathbb{C}\right\}
$$

(and $-\Delta_{0}^{[\delta]}$ acting on $D_{\delta}$ as $-\Delta_{0}^{*}$ ).

Clearly, the full domain of $-\Delta_{0}^{[\delta]}$ is then equal to

$$
\left.\left\{\psi \in \mathscr{D}\left(-\Delta_{0}^{*}\right):\left\langle-\Delta_{0} \phi \mid \psi\right\rangle_{\mathscr{L}}=\left\langle\phi \mid-\Delta_{0}^{*} \psi\right\rangle_{\mathscr{L}}, \forall \phi \in D_{\delta}\right\rangle\right\}
$$

A simple integration by parts shows that this is equal to

$$
\left\{\psi \in \mathscr{D}\left(-\Delta_{0}^{*}\right): \lim _{\varrho \rightarrow 0}\left(\psi_{\delta} \varrho \psi^{\prime}-\psi \varrho \psi_{\delta}^{\prime}\right)=0\right\}
$$

where $\psi_{\delta}=\psi_{+}+\delta \psi_{-}$.

One may use the asymptotic formula (for $z \downarrow 0$ )

$$
K_{0}(z)=-\ln (z / 2)-C+O\left(z^{2} \ln (z)\right),
$$

where $C$ is Euler's constant to see that

$$
\psi_{\delta}=(1+\delta)(-\ln (\varrho / 2)-C)+(1-\delta) i \pi / 4+O\left(\varrho^{2} \ln \varrho\right)
$$

Exchanging $\delta$ for the real label $q$ (ranging over $[0, \infty)$ ) defined by

$$
q=\exp \left(-i \frac{\pi}{4} \frac{(1-\delta)}{(1+\delta)}\right)
$$

(by convention, we take $q=0$ when $\delta=-1$ ) this may be written conveniently as

$$
\psi_{\delta}= \begin{cases}\operatorname{const}(\ln (q \varrho / 2)+C)+O\left(\varrho^{2} \ln \varrho\right) & \text { if } q \in(0, \infty) \\ \operatorname{const}+O\left(\varrho^{2} \ln \varrho\right) & \text { if } q=0 .\end{cases}
$$


We may thus finally re-express the domain of $-\Delta_{0}^{[\delta]}$ (which we now rename $-\Delta_{0}^{(q)}$ ) as

$$
\left\{\psi \in \mathscr{D}\left(-\Delta_{0}^{*}\right): \lim _{\varrho \rightarrow 0}\left((\ln (q \varrho / 2)+C) \varrho \psi^{\prime}-\psi\right)=0\right\}
$$

in case $q \in(0, \infty)$, and

$$
\left\{\psi \in \mathscr{D}\left(-\Delta_{0}^{*}\right): \lim _{\varrho \rightarrow 0} \varrho \psi^{\prime}=0\right\}
$$

in case $q=0$. (Note that to show that the terms $O\left(\varrho^{2} \ln \varrho\right.$ ) do not affect this limit, one may use Property (ii) above.) Finally, exchanging $q$ for $R$ by (1.7) (see Note $\langle 4\rangle$ ) one sees that we have arrived at a rigorous justification of the boundary condition (2.3).

To end this subsection, we remark that, quite generally, a symmetric operator $A$ will have self-adjoint extensions only when its deficiency indices are equal and, in the case of deficiency indices $\langle n, n\rangle$, these extensions are necessarily labelled by the unitary group $U(n)$ (see e.g. Sect. X.1 in [18]). If in this general case, one denotes the extension corresponding to the label $\alpha \in U(n)$ by $A^{\alpha}$, then it follows easily, e.g. from the results in Sect. X.1 in [18], that, given any convergent sequence

$$
\alpha_{j} \rightarrow \alpha
$$

in the labelling group, one will have convergence of the operators $A^{\alpha_{j}}$,

$$
A^{\alpha_{j}} \rightarrow A^{\alpha}
$$

in the sense that, for all $t \in \mathbb{R}$ and vectors $\psi$ in the Hilbert space, the sequence $\exp \left(A^{\alpha_{j}} t\right) \psi$ converges to $\exp \left(A^{\alpha} t\right) \psi$. Adopting this notion of convergence, and applying this result to our case of interest (minus the Laplacian on a cone with $\kappa \leqq 1$ ), where $U(n)$ becomes a copy of the circle, and our parameters $q$ and $R$ become different ways of relabelling points on the circle - one sees that $-\Delta^{(q)} \rightarrow-\Delta^{0}$ as $q \rightarrow 0$ and $-\Delta^{R} \rightarrow-\Delta^{0}$ as $R \rightarrow 0$ (in other words, $-\Delta^{(q)} \rightarrow-\Delta^{0}$ also as $q \rightarrow \infty$ ). These remarks justify the point made at the end of Note $\langle 4\rangle$. (See also [42] for further discussion.)

A1.4. The remaining statements (after Eq. (2.3)) in Sect. 2 may be given a suitable rigorous justification by e.g. supplementing the methods of $[17,18]$ with appropriate aspects of the theory of ordinary differential equations - see e.g. [27]. (Useful heuristic techniques for arriving at the complete sets of eigenfunctions ((2.4) for $q=0,(2.9)$ for $q \geqq 0$ ) and for quickly determining the correct normalization factors may be found, e.g. in Chap. 7 of [19]. See also [25].) In particular the "complete sets of eigenfunctions" (2.4) for $q=0,(2.9)$ for $q \geqq 0$ may be shown to provide spectral representations for each of the self-adjoint extensions $-\Delta^{(q)}$. Thus, in case $q=0(R=0)$, the map

$$
\begin{gathered}
V^{(0)}: L^{2}\left(\mathbb{R}^{+} \times \mathbb{S}, \varrho d \varrho d \theta\right) \rightarrow \bigoplus_{m \in \mathbb{Z}} L^{2}\left(\mathbb{R}^{+}, p d p\right) \\
\psi \mapsto \bigoplus_{m \in \mathbb{Z}} \tilde{\psi}_{m},
\end{gathered}
$$

where

$$
\tilde{\psi}_{m}(p)=\int_{0}^{\infty} \int_{0}^{2 \pi} \psi_{m, p}(\varrho, \theta) \psi(\varrho, \theta) \varrho d \varrho d \theta
$$


and, in case $q \geqq 0(R \geqq 0)$, the map

$$
\begin{aligned}
V^{(q)}: L^{2}\left(\mathbb{R}^{+} \times \mathbb{S}, \varrho d \varrho d \theta\right) & \rightarrow \underset{m \in \mathbb{Z}}{\bigoplus} L^{2}\left(\mathbb{R}^{+}, p d p\right) \oplus \mathbb{C} \\
\psi & \mapsto \underset{m \in \mathbb{Z} \backslash\{0\}}{\bigoplus} \tilde{\psi}_{m} \oplus \tilde{\psi}_{0}^{(q)} \oplus \tilde{\psi}_{\text {bound }},
\end{aligned}
$$

where $\tilde{\psi}_{0}^{(q)}(p)$ is defined by (A1.2) with $\psi_{0, p}(\varrho)$ replaced by $\psi_{0, p}^{(q)}(\varrho)$ (cf. (2.7)) and (cf. (2.6))

$$
\tilde{\psi}_{\text {bound }}=\int_{0}^{\infty} \int_{0}^{2 \pi} \psi_{\text {bound }}(\varrho) \psi(\varrho, \theta) \varrho d \varrho d \theta
$$

are isomorphisms which intertwine $-\Delta^{(q)}$ with a multiplication operator

$$
V^{(q)}\left(-\Delta^{(q)}\right) V^{(q) *}=\bigoplus_{m \in \mathbb{Z}} p^{2} \oplus\left(-q^{2}\right)
$$

( $p^{2}$ the multiplication operator on $L^{2}\left(\mathbb{R}^{+}, p d p\right)$.)

Note that (i) We have chosen all the eigenfunctions to be real. (ii) The sums and integrals ((A1.2) etc.) are to be understood with a suitable notion of $L^{2}$-convergence. (iii) In the cases $q=0$ and/or $m \neq 0$, the $\varrho$-integral in (A1.2) is just the well-known Hankel transform. For $q \neq 0, m=0$, the eigenfunctions $\psi_{0, e}^{(q)}$ thus lead to a kind of generalized Hankel transform.

\section{A2. "Symplectic Ideology"}

The main purpose of this appendix is to give a suitable notion of "acceptable global time evolution" in the case of Eq. (1.2) on the static spacetime around an idealized cosmic string $(\mathscr{M}, g)(1.1)\langle 1\rangle$. There may be several ways to do this.

We choose a way that relies on the finite-propagation-speed property of hyperbolic equations such as (1.2). For any initial data

$$
(\varphi, \pi) \in C_{0}^{\infty}\left(\mathbb{R}^{+} \times \mathbf{S} \times \mathbb{R}\right) \times C_{0}^{\infty}\left(\mathbb{R}^{+} \times \mathbf{S} \times \mathbb{R}\right)
$$

there will be a time-interval $\delta_{\varphi, \pi}$ (the minimum distance from the support of the data to the cosmic string, i.e. the minimum time for the data to reach the string) within which the Cauchy problem will be well posed in the sense that there will be a unique solution $\phi$ in the time-interval $\delta_{\varphi, \pi}$ (with Cauchy data remaining in $\left.C_{0}^{\infty}\left(\mathbb{R}^{+} \times \mathbf{S} \times \mathbb{R}\right) \times C_{0}^{\infty}\left(\mathbb{R}^{+} \times \mathbf{S} \times \mathbb{R}\right)\right)$ which has these Cauchy data (i.e. $\varphi=\phi$, $\pi=\dot{\phi}$ ) at time zero. We call the map from such data at time zero to data at time $t$ the "local time evolution" $\mathscr{T}_{\text {loc }}(t)$, where it is understood that $(\varphi, \pi)$ will be in the domain of $\mathscr{T}_{\text {loc }}(t)$ only when $|t|<\delta_{\varphi, \pi}$. Note that $\mathscr{T}_{\text {loc }}(t)$ will then be a "local group" in the sense that

$$
\mathscr{T}_{\text {loc }}\left(t_{1}\right) \mathscr{T}_{\text {loc }}\left(t_{2}\right)=\mathscr{T}_{\text {loc }}\left(t_{1}+t_{2}\right)
$$

will hold on the intersection of the domains of the left- and right-hand sides and it will also preserve the symplectic form (3.7) on its domain at each $t$,

$$
\sigma\left(\mathscr{T}_{\text {loc }}(t)\left(\varphi_{1}, \pi_{1}\right) ; \mathscr{T}_{\text {loc }}(t)\left(\varphi_{2}, \pi_{2}\right)\right)=\sigma\left(\left(\varphi_{1}, \pi_{1}\right) ;\left(\varphi_{2}, \pi_{2}\right)\right) .
$$

We shall define an acceptable global time-evolution to consist of a global symplectic time-evolution group $\mathscr{T}(t)$ which extends $\mathscr{T}_{\text {loc }}(t)$. More precisely, it consists of an extension $(D, \hat{\sigma})$ of the symplectic space

$$
\left(C_{0}^{\infty}\left(\mathbb{R}^{+} \times \mathbb{S} \times \mathbb{R}\right) \times C_{0}^{\infty}\left(\mathbb{R}^{+} \times \mathbf{S} \times \mathbb{R}\right), \sigma\right)
$$


and a symplectic group $\mathscr{T}(t)$ mapping $D$ to $D$ such that

$$
\mathscr{T}(t)(\varphi, \pi)=\mathscr{T}_{\text {loc }}(t)(\varphi, \pi)
$$

whenever the right-hand side is defined. Finally, in line with our interest in translationally invariant boundary conditions, we shall say that such a global symplectic extension $(D, \hat{\sigma}, \mathscr{T}(t))$ is translationally invariant (in $z)$ if $D$ also admits an extension

$$
Z\left(z^{\prime}\right):(\varphi(\varrho, \theta, z), \pi(\varrho, \theta, z)) \mapsto\left(\varphi\left(\varrho, \theta, z+z^{\prime}\right), \pi\left(\varrho, \theta, z+z^{\prime}\right)\right)
$$

of the group of $z$-translations on $\left(C_{0}^{\infty}\left(\mathbb{R}^{+} \times \mathbf{S} \times \mathbb{R}\right) \times C_{0}^{\infty}\left(\mathbb{R}^{+} \times \mathbf{S} \times \mathbb{R}\right), \sigma\right)$ whose elements commute with the $\mathscr{T}(t)$ 's.

We claim that, for each $q$, the space $\left(D_{\mu}^{(q)}, \hat{\sigma}\right)$ with $\left(D_{\mu}^{(q)}\right.$ as in (3.5) and $\hat{\sigma}$ defined by (3.7)) together with $\mathscr{T}_{\mu}^{(q)}(t)$ (3.6) constitutes a (translationally-invariant) global symplectic time evolution in the sense just defined. To check this, it is easy to see that $\mathscr{T}_{\mu}^{(q)}(t)$ maps $D_{\mu}^{(q)} \rightarrow D_{\mu}^{(q)}$, preserves the symplectic form $\hat{\sigma}$ (and commutes with $z$-translations). It thus only remains to show that $\mathscr{T}_{\mu}^{(q)}(t)$ extends $\mathscr{T}_{\text {loc }}(t)$ in the sense of (A2.1). This may be done by showing that, on

$$
C_{0}^{\infty}\left(\mathbb{R}^{+} \times \mathbf{S} \times \mathbb{R}\right) \times C_{0}^{\infty}\left(\mathbb{R}^{+} \times \mathbf{S} \times \mathbb{R}\right),
$$

both locally solve the Cauchy problem in an $L^{2}$-sense, and proving the uniqueness of such $L^{2}$-solutions. (We omit the details.)

We remark that, in the construction of Sect. 3, D could have been chosen slightly differently. For example, we could have chosen the slightly smaller domain in $\mathscr{H} \oplus \mathscr{H}$,

$$
\hat{D}_{\mu}^{(q)}=\mathscr{D}\left(\left|A_{\mu}^{(q)}\right|\right) \oplus \mathscr{D}\left(\left|A_{\mu}^{(q)}\right|^{1 / 2}\right)
$$

One easily checks that this is equally an invariant domain for $\mathscr{T}(t)$, and still contains

$$
C_{0}^{\infty}\left(\mathbb{R}^{+} \times \mathbf{S} \times \mathbb{R}\right) \times C_{0}^{\infty}\left(\mathbb{R}^{+} \times \mathbf{S} \times \mathbb{R}\right) .
$$

Clearly $\left(\hat{D}_{\mu}^{(q)}, \hat{\sigma}, \mathscr{T}_{\mu}^{(q)}(t)\right)$ and $\left(D_{\mu}^{(q)}, \hat{\sigma}, \mathscr{T}_{\mu}^{(q)}(t)\right)$ (and more generally, any pair of global symplectic extensions when one is an extension of the other) describe the same choice of boundary conditions. We shall say they do not differ in essence.

We leave as an open question whether there exist translationally invariant "exotic" global symplectic extensions of

$$
\left(C_{0}^{\infty}\left(\mathbb{R}^{+} \times \mathbf{S} \times \mathbb{R}\right) \times C_{0}^{\infty}\left(\mathbb{R}^{+} \times \mathbf{S} \times \mathbb{R}\right), \sigma, \mathscr{T}_{\text {loc }}(t)\right)
$$

which differ in essence from all those constructed in Sect. 3 (i.e. which do not arise from self-adjoint extensions of $A$ (3.2)). (Note: Throughout the remainder of the paper, we shall proceed as if there are no such exotic extensions.) As a final remark, note that, although we have restricted consideration in Sect. 3 and this appendix to translationally invariant extensions of the dynamics, the approach we have adopted (and the issues we have raised) are not restricted in principle to these cases (cf. Note $\langle 17\rangle$ ). In fact, our approach should also further generalize to a wide class of problems involving linear stationary hyperbolic systems with boundaries - such as Eq. (1.2) on a wide class of stationary spacetimes which fail to be globally hyperbolic. 


\section{Notes}

1. Planckian units are used throughout $\hbar=c=G=1$.

2. In the non-relativistic Schrödinger equation, we set the mass $\mu=\frac{1}{2}$.

3. For convenience, we drop a factor of $\kappa$ from the volume element.

4. We shall also set, by convention, $q=0$ when $R=0$. In Sect. 1 to 4 , we shall find it convenient to use $q$ (related to $R$ by Eq. (1.7) and this convention and also taking its values in $[0, \infty)$ ) rather than $R$ as a label for our self-adjoint extensions. (We shall indicate $q$-labels by the use of round brackets. Thus $-\Delta^{(3)}$ denotes the extension corresponding to $q=3$ units of inverse length, while $-\Delta^{3}$ denotes the extension corresponding to $R=3$ units of length. Note that $-\Delta^{(0)}=-\Delta^{0}$.)

In general, the $q$ label is useful when one is interested in the energy of the bound state, $\left(-q^{2}\right)$, while the $R$ label is appropriate in the application discussed in Sect. 5 to fitting the dynamics on rounded off cones etc., since - as we explain there - it corresponds to the scattering length of the true interaction. Note that the discontinuity at $R=0$ in the relation between $q$ and $R$ is physically spurious: One should really think of the parameter space labelling the different self-adjoint extensions of $-\Delta$ as topologically a circle with $q=0$ and $q=\infty$ (equivalently $R=0$ and $R=\infty$ ) identified. In fact (see the last remarks in Sect. A1.3 and [42]) one has (with a suitable notion of convergence) $-\Delta^{(q)} \rightarrow-\Delta^{(0)}$ as $q \rightarrow \infty$ (and also $-\Delta^{R}$ $\rightarrow-\Delta^{0}$ as $R \rightarrow \infty$ ).

5. Modulo possible fine points which we ignore here (see e.g. the relevant discussion in Sect. 3 of [22]) for each $R$, the ground state which we construct here will be uniquely determined up to the possible addition of a non-vanishing onepoint function. In particular, the two-point function (4.9) will be unique.

6. (See also Note $\langle 20\rangle$ ) See e.g. [45]. Note that our definition of two-dimensional scattering length (see [42] for a full discussion) differs from that discussed in [28], which was introduced in $[46,47]$. In particular, the two-dimensional scattering length of $[28,46,47]$ is dimensionless, while our notion has the dimensions of length.

7. Here, one may ignore gravity, and view the components of the electromagnetic vector potential (say in the Lorentz gauge) simply as four massless scalar waves with a potential - or "local mass" term inside the string core.

8. Note that it is essential for this result that $0<\kappa \leqq 1$. For $\kappa>1$, by Theorem X.10 in [18], there will be further (non-circularly symmetric) self-adjoint extensions of $-\Delta-$ with $-\Delta_{m}$ failing to be essentially self-adjoint whenever $|m|<\kappa$. Note that, according to the principles discussed in [42] and Sect. 5 here, these results should be relevant to a cone which was rounded-off in a non-circularly symmetric way (especially if the potential term in (1.10) failed to be circularly symmetric) or to a cosmic string which interacted with the Klein-Gordon field in a non-cylindrically symmetric way inside the string radius. For $\kappa \leqq 1$ one would expect physical effects at large length scales to be quite insensitive to such asymmetries. For $n<\kappa \leqq n+1$ one would instead expect large scale effects (such as the low-energy scattering amplitudes) to be sensitive to the shape of the interaction through, and in fact to strongly feel, its Fourier components for $m \leqq n$. 
9. (See also Note $\langle 20\rangle$.) The situation here is to be contrasted with the self-adjoint extensions of minus-the-Laplacian on Euclidean 3-space with a point removed. Here again the non-uniqueness lies in the spherically symmetric sector where (cf. [18], Appendix to Sect. X.1, Example 4) the problem is equivalent to the problem of $-d^{2} / d x^{2}$ on ( $L^{2}$-functions on) the half line. Here, it may easily be seen that there is a one-parameter family of self-adjoint extensions, "half" of which are positive and "half" having bound states.

10. In one important sense, the discussion of possible boundary conditions in the present paper lacks full generality: For quantum mechanics on the cone (Sect. 2), the set of self-adjoint extensions of minus-the-Laplacian will not be appropriate for modelling physical situations in which the wave function "loses amplitude" (i.e. particles are absorbed) at whatever is being modelled by the conical singularity. Such situations could be modelled by enlarging our considerations to include certain non-self adjoint (but say accretive, see e.g. [18]) extensions (cf. the discussion in [28]) corresponding to certain non-unitary time-evolution groups. Similarly, by restricting ourselves in Sects. 3 and A 2 to symplectic extensions of the local time evolution $\mathscr{T}_{\text {loc }}(t)$, we are implicitly restricting the physical situation being modelled to waves which interact elastically with the cosmic string. Our discussion of the quantum field theoretic problem in Sects. 4 and 5 is similarly limited to elastic processes.

11. The equation $i \partial \psi / \partial t=\left(\mu^{2}-\Delta\right)^{1 / 2}$ is the one-particle dynamics for the relativistic quantum field theory of $\left(\partial^{2} / \partial t^{2}-\Delta+\mu^{2}\right) \psi=0$ on the cone. More insight into this will be obtained in Sect. 4 where we discuss the quantized Klein-Gordon equation on the $1+3$ dimensional spacetime where the space is the product of the two-dimensional cone with the real line. Note however that (as in flat spacetime) certain new technicalities will arise for the true two-dimensional cone if the mass is zero (e.g. $C_{0}^{\infty}\left(\mathbb{R}^{+} \times \mathbf{S}\right)$ will no longer belong to $\mathscr{D}\left(\left(-\Delta^{(0)}\right)^{-1 / 2}\right)$ cf. Note $\left.\langle 19\rangle\right)$. Also the case $q=\mu$, where there is a discrete eigenvector with zero energy would require special treatment.

12. To prove that, in the case $\kappa=1$, the extension for $q=0$ (i.e. since it is the unique positive extension, the Friedrichs extension) of

$$
-\Delta \text { on } C_{0}^{\infty}\left(\mathbb{R}^{+} \times \mathbf{S}\right) \subset L^{2}\left(\mathbb{R}^{+} \times \mathbf{S}, \varrho d \varrho d \theta\right)
$$

(equivalently of $-\Delta$ on $C_{0}^{\infty}\left(\mathbb{R}^{2} \backslash\{0\}\right) \subset L^{2}\left(\mathbb{R}^{2}\right)$ ) is equal (under the same equivalence) to the unique self-adjoint extension of $-\Delta$ on $\left.C_{0}^{\infty}\left(\mathbb{R}^{2}\right) C L^{2}\left(\mathbb{R}^{2}\right)\right)$ in $L^{2}\left(\mathbb{R}^{2}\right)$ observe that $C_{0}^{\infty}\left(\mathbb{R}^{2}\right)$ contains the (by the results of Sect. A1) core $D_{-1} \otimes D_{\mathbf{S}}$ (finite sums of products) for $-\Delta^{(0)}$, where $D_{-1}$ consists of $C^{\infty}$ functions $(\psi)$ of $\varrho$ which vanish at sufficiently large $\varrho$ and satisfy $\lim _{\varrho \rightarrow 0} \varrho \partial \psi / \partial \varrho=0$.)

13. It is tempting to expect that, in the case of $\kappa=1,-\Delta^{(q)}$, for $q \geqq 0$, would be equivalent to the Schrödinger operator with a " $\delta$-function potential" at the origin. Actually, the notion of a $\delta$-function potential can be given a direct mathematical meaning, e.g. using the notion of quadratic forms on Hilbert spaces, only in one dimension, see e.g. Example 3 in Sect. X.2 of [18]. (Note that the one-dimensional case, i.e. $-\partial^{2} / \partial x^{2}$ on $C_{0}^{\infty}(\mathbb{R} \backslash\{0\}) C L^{2}(\mathbb{R})$ actually has deficiency indices $\langle 2,2\rangle$ so there are also other kinds of self-adjoint extensions in this case, see [28].) Nevertheless, in two and three dimensions (which are the only other dimensions 
for which $-\Delta$ on $C_{0}^{\infty}\left(\mathbb{R}^{n} \backslash\{0\}\right) \subset L^{2}\left(\mathbb{R}^{n}\right)$ fails to be essentially self-adjoint) one may think of the different self-adjoint extensions of minus-the-Laplacian on $\left(C_{0}^{\infty}\right.$ functions on) $\mathbb{R}^{n} \backslash\{0\}$ as corresponding to the addition to minus-the-usualLaplacian of some sort of suitably renormalized $\delta$-function potential. This idea is extensively discussed in the monograph [28] where the emphasis, however, is more on the three dimensional case. Here we would remark that, from this point of view, the two-dimensional case appears (in contrast to the three dimensional case, see Note $\langle 9\rangle$ ) rather strange since the existence of a "bound state" for all $q>0$ seems to say that, in two-dimensions, the only non-trivial renormalized $\delta$-function potentials are attractive. Light will be shed on this matter in Sect. 5.2, where we point out that, in using $-\Delta^{(q)}$ for some non-zero $q$ to approximate the large-scale behaviour of a positive potential of small support, the bound state should be considered as spurious. (See also [42]).

14. There are really two distinct scattering problems one can reasonably pose for quantum mechanics on the cone according to what one chooses to regard as the appropriate "comparison free-dynamics." Given the dynamics $\exp \left(i \Delta_{\kappa}^{(q)} t\right)$ corresponding to the choice of the $q^{\text {th }}$ self-adjoint extension of the Laplacian on the cone of deficit angle $\delta=2 \pi(1-\kappa)$, one may compare this either

(i) with the dynamics $\exp \left(i \Delta_{\kappa}^{(0)} t\right)$ corresponding to the choice of the Friedrichs extension on the same cone (i.e. with the same $\kappa$ ) or

(ii) the free dynamics on flat (two-dimensional) space (i.e. by Note $\langle 12\rangle$ with $\left.\exp \left(i \Delta_{k=1}^{(0)} t\right)\right)$.

In case (ii), it is necessary to geometrically identify the cone with flat space. An obvious way to do this is to identify points with the same $(r, \theta)$ coordinates. Note that while this is of course not a global isometry, it does preserve (up to an unimportant numerical factor) the volume element $\varrho d \varrho d \theta$ and thus induces an isomorphism between the resulting $L^{2}$-spaces.

The natural mathematical problems to isolate concern the existence and properties of two different types of wave operators:

and

$$
\omega_{\kappa}^{(q) \pm}=s-\lim _{t \rightarrow \pm \infty} \exp \left(-i \Delta_{\kappa}^{(q)} t\right) \exp \left(i \Delta_{\kappa}^{(0)} t\right)
$$

$$
\Omega_{\kappa}^{(0) \pm}=s-\lim _{t \rightarrow \pm \infty} \exp \left(-i \Delta_{\kappa}^{(0)} t\right) \exp \left(i \Delta_{1}^{(0)} t\right),
$$

where $\Delta_{1}^{(0)}$ is the usual Laplacian on flat space and in $(* *)$, the identification of the cone and flat space Hilbert spaces as described above is understood. The wave operators $\omega_{\kappa}^{(q) \pm}$ clearly correspond to comparison (i) while the general comparison (ii) above would have to do with the composition

$$
\Omega_{\kappa}^{(q) \pm}=\omega_{\kappa}^{(q) \pm} \Omega_{\kappa}^{(0) \pm}
$$

of these two different types of wave operators.

We note that the "change in the phase shift" considered in this paper refers to comparison (i) (i.e. the $\omega$ 's). On the other hand, the phase shift introduced by Deser and Jackiw [14] would appear to be related to Comparison (ii) in the Friedrichs extension case, i.e. to the wave operators $\Omega_{\kappa}^{(0) \pm}$.

It would be an interesting problem to gain mathematical control over the two types of wave operators $(*)$ and $(* *)$. We remark that, in the case of zero deficit angle $(\kappa=1)$ the scattering theory associated with $(*)$ is discussed in Chap. I.5 of [28]. 
15. Levinson's theorem (which is usually proved for suitable classes of potentials for Schrödinger operators in three dimensions, see e.g. [39,40]) states that if one normalizes the phase shift to vanish at infinite momentum, then the phase shift at zero momentum is equal to $\pi$ times the number of bound states.

16. In this note, we wish to draw attention to some interconnections between the boundary-condition problem for the Laplacian on a cone and the problem of the classical motion of a non-relativistic particle on a cone or on the space around an idealized cosmic string. As Linet (in the electrostatic case) [29] and Smith [4] (see also [25]) have recently pointed out (in the context of motion near a cosmic string) one might expect such a particle (located say at $\mathbf{x}$ with coordinates $\varrho, \theta, z$ ) to feel a self-force due to the distortion of its own gravitational potential. At points $\mathbf{x}^{\prime}$ other than $\mathbf{x}$, this potential, which we denote $G_{M}\left(\mathbf{x}, \mathbf{x}^{\prime}\right)$ will satisfy the appropriate Poisson equation. In the case of motion near an idealized straight cosmic string (1.1) this will be governed by

$$
-\left(\Delta+\partial^{2} / \partial z^{2}\right) G_{M}\left(\mathbf{x}, \mathbf{x}^{\prime}\right)=4 \pi M \delta^{(3)}\left(\mathbf{x}, \mathbf{x}^{\prime}\right),
$$

where $M$ is the mass of the test particle (cf. the operator $A_{\mu}$ of Sect. 3 in case $\mu=0$ ).

The self-potential $V_{M}$ for a particle of mass $M$ is then reasonably calculated, say in a point-splitting renormalization scheme, to be given by

$$
V_{M}(x)=-\left(M^{2} / 2\right) \lim _{\mathbf{x}^{\prime} \rightarrow \mathbf{x}}\left(G_{M}\left(\mathbf{x}, \mathbf{x}^{\prime}\right)-G_{0}\left(\mathbf{x}, \mathbf{x}^{\prime}\right)\right),
$$

where $G_{0}$ is the solution of Poisson's equation one would obtain if the locally flat geometry around $\mathbf{x}$ were viewed as embedded in a globally flat space instead of the physical conical space. Similarly, in the case of an electrically charged test particle, the electrostatic potential $G_{e}$ will be governed by the same equation $(*)$ with $e$ (the charge of the test particle) in place of $M$, while for the electrostatic self-potential $V_{e}$, one must replace $-M^{2} / 2$ in (**) by $e^{2} / 2$. Here, we wish to make two remarks (see also [25]). Firstly, in calculating the Green's functions $G_{M}$ and $G_{e}$, (i.e. the inverse of the appropriate Laplacian) one must make a choice of boundary condition at the conical singularity (or string). In doing this, Smith and Linet appear to implicitly choose the Friedrichs extension of their Laplacian, obtaining the values (for small string-mass per unit length $\lambda=(1 / 4)(1-\kappa)$

$$
V_{M}(\mathbf{x})=\pi \lambda M^{2} / 4 r, \quad V_{e}(\mathbf{x})=-\pi \lambda e^{2} / 4 r .
$$

However, in principle, one could take for $G_{M}$ (or $G_{e}$ ) the inverse of any of the $(z$-translationally invariant) self-adjoint extensions of the differential operator in $(*)$. In other words, one can solve $(*)$ for any of the inequivalent boundary conditions $(1.8) /(2.3)$. For each choice, one expects, of course a different result for $V_{M}\left(\right.$ or $\left.V_{e}\right)$.

Secondly, it is problematic whether any of the resulting self-potential-formulae will be a good approximation to the true self-potential due to a true string. One, of course, expects these latter to be governed, in the gravitational case by:

$$
-\left(\Delta_{\tilde{h}}\right) G_{M}^{\text {true }}\left(\mathbf{x}, \mathbf{x}^{\prime}\right)=4 \pi M \delta^{(3)}\left(\mathbf{x}, \mathbf{x}^{\prime}\right)
$$

and, in the electrostatic case (say for a superconducting cosmic string, cf. Sect. 5.2) by

$$
-\left(\Delta_{\tilde{h}}+\tilde{V}\right) G_{e}^{\mathrm{true}}\left(\mathbf{x}, \mathbf{x}^{\prime}\right)=4 \pi e \delta^{(3)}\left(\mathbf{x}, \mathbf{x}^{\prime}\right)
$$


We remark that, on the basis of the principle result in Sect. 5.1, one might expect the true gravitational self-force (or the true electrostatic self-force in the case of a non-superconducting cosmic string) to be well approximated by taking the Friedrichs extension ("usual" boundary condition) in the corresponding idealized problem. On the other hand, on the basis of the discussion in Sect. 5.2, one might expect the true electrostatic self force due to a superconducting string to be best approximated by taking the boundary condition for the appropriate fitted $R$-value. However, it is not immediately clear to what extent either of these approximations would be "believable" since (like the quantum energy-momentum tensor, cf. the discussion at the end of Sect. 5.2) the self-force involves both large and small scales. Concerning believability for the case $R=0$, Davis and Perkins $[62,63]$ have recently computed the corrections to the electrostatic self-force on a test charge near a cosmic string due to the rounding off of the metric inside the string (but without considering any possible $\widetilde{V}$ term which - as we point out here ought to be included in the superconducting case) and find that the value is very close to that for an idealized string with $R=0$ boundary conditions once one is beyond two to three string radii. Work is in progress [53] on calculating the selfforce for an idealized string with non-zero $R$ values, and on the question of the believability of this latter quantity in modelling the effect of the local photon mass term $\widetilde{V}$ on the self-potential of a test charge near a superconducting string.

Finally, the question arises whether, and when (depending on the physical application), it might be appropriate to include the potential $V_{M}$ (or $V_{e}$ or both) in the Schrödinger equation for the quantum motion of a test particle moving on the idealized-string spacetime. (Also, the obvious analogue question arises for a 2-dimensional cone.) On top of the choice of self-adjoint extension needed to define $V_{M}$ and $V_{e}$ as discussed above, one would then presumably have to face a second self-adjoint extension problem for the resulting Schrödinger operator (say for the string problem)

$$
-\left(\Delta+\partial^{2} / \partial z^{2}\right)+V_{e}+V_{M}
$$

In the case where one takes the Friedrichs extension in the first self-adjointextension problem (i.e. with the "usual" formula for the self-force), and a particular choice of self-adjoint extension (corresponding to finiteness of the wave function at the origin) in the second problem, the scattering theory of the above Schrödinger operator has recently been treated by Gibbons et al. [41].

17. Rigorously, let $D_{1} \subset \mathscr{H}_{1}=L^{2}\left(\mathbb{R}^{+} \times \mathbb{S}, \varrho d \varrho d \theta\right)$ be any core for $-\Delta^{(q)}$ and $D_{2} \subset \mathscr{H}_{2}$ $=L^{2}(\mathbb{R}, d z)$ any core for $-\partial^{2} / \partial z^{2}$ (e.g. $\left.D_{2}=C_{0}^{\infty}(\mathbb{R})\right)$. Then, by Theorem VIII.33 in $[17]$,

$$
\mu^{2} \mathbb{I} \otimes \mathbb{I}+\left(-\Delta^{(q)}\right) \otimes \mathbb{I}+\mathbb{I} \otimes\left(-\partial^{2} / \partial z^{2}\right)
$$

is essentially self adjoint on the domain $D_{1} \otimes D_{2}$ consisting of finite sums of products of elements of $D_{1}$ and $D_{2}$ in $\mathscr{H}_{1} \otimes \mathscr{H}_{2}$. A suitable way to define $A_{\mu}^{(q)}$ is to take it to be the closure of this operator. We remark that $A_{\mu}$ on $C_{0}^{\infty}\left(\mathbb{R}^{+} \times \mathbf{S} \times \mathbb{R}\right)$

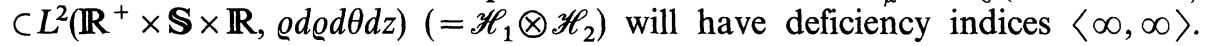
However, it is easy to convince oneself that amongst all the resulting self-adjoint extensions, only the one-parameter family $\left\{A_{\mu}^{(q)}: q \in[0, \infty)\right\}$ will be translationally invariant in $z$ (i.e. commute with $z$-translations).

18. A full argument for boundedness of the values of the Klein-Gordon field could presumably be made by combining (3.7) with suitable Sobolev estimates (cf. 
$[20,21])$. Note that for the "borderline" cases $\mu=0, q=0$, and $\mu=q(2.7)$ the situation becomes delicate and would require a special discussion.

19. That $C_{0}^{\infty}\left(\mathbb{R}^{+} \times \mathbb{S} \times \mathbb{R}\right) \subset \mathscr{D}\left(\left|A_{0}^{(0)}\right|^{-1 / 2}\right)(4.10)$ may be established by combining the following three results. Note, in the rest of this note, we change notation so that $A_{\kappa}$ denotes $A_{0}^{(0)}$ on the cone with deficit angle $2 \pi(1-\kappa)$. We shall also take for granted, in the case of $\kappa=1$, the obvious identification of the cone with flat twodimensional space with a point removed, and thus e.g. identify $C_{0}^{\infty}\left(\mathbb{R}^{+} \times \mathbf{S}\right)$ in $L^{2}\left(\mathbb{R}^{+} \times \mathbb{S}, \varrho d \varrho d \theta\right)$ with $C_{0}^{\infty}\left(\mathbb{R}^{2} \backslash\{0\}\right)$ in $L^{2}\left(\mathbb{R}^{2}\right)$ etc., switching between polar and Cartesian coordinates without comment.

(i) $A_{1}$ may be identified with minus the ordinary three-dimensional Laplacian $\hat{A}_{1}$, i.e. the closure of minus the (essentially self-adjoint) Laplacian on $C_{0}^{\infty}\left(\mathbb{R}^{3}\right)$ in $L^{2}\left(\mathbb{R}^{3}\right)$. (This was proven in Note $\langle 12\rangle$.)

(ii)

$$
C_{0}^{\infty}\left(\mathbb{R}^{+} \times \mathbb{S} \times \mathbb{R}\right) \subset C_{0}^{\infty}\left(\mathbb{R}^{3}\right) \subset \mathscr{D}\left(\hat{A}_{1}^{-1 / 2}\right) .
$$

This may be proven by standard Fourier transform methods. (iii) $A_{1}^{-1 / 2}$ is densely defined and $\mathscr{D}\left(A_{1}^{-1 / 2}\right) \subset \mathscr{D}\left(A_{\kappa}^{-1 / 2}\right), \kappa \leqq 1$.

It remains only to prove (iii). We do this by geometrically identifying the cones with different $\kappa$ values as in Note $\langle 14\rangle$ and observing from (1.6) that for $\kappa \leqq 1$, we then have the inequality

$$
\left\langle\psi \mid A_{\kappa} \psi\right\rangle \geqq\left\langle\psi \mid A_{1} \psi\right\rangle
$$

for all $\psi$ in the common core (see Sect. A1.3) $D_{-1} \otimes D_{\mathrm{S}}$. From this, we may conclude that

$$
\left\|A_{\kappa}^{1 / 2} \psi\right\| \geqq\left\|A_{1}^{1 / 2} \psi\right\|, \quad \forall \psi \in \mathscr{D}\left(A_{\kappa}^{1 / 2}\right),
$$

whereupon $A_{1}^{1 / 2} A_{\kappa}^{-1 / 2}$ is bounded, and hence by taking adjoints $A_{\kappa}^{-1 / 2} A_{1}^{1 / 2}$ is also bounded. This implies $\left\|A_{\kappa}^{-1 / 2} \psi\right\| \leqq\left\|A_{1}^{-1 / 2} \psi\right\|, \forall \psi \in \mathscr{D}\left(A_{1}^{-1 / 2}\right)$ which implies (iii). (Cf. Lemma A4.9 in [34]).

20. (See also Notes $\langle 6\rangle$ and $\langle 9\rangle$.) We briefly sketch here the relevant threedimensional notions - assuming for simplicity a flat three dimensional space attempting to bring out the analogy with the treatment of the two-dimensional case given in the text. In this (flat) three dimensional case, the analogue of our selfadjoint extension problem concerns the self-adjoint extensions of $-\Delta$ on $C_{0}^{\infty}\left(\mathbb{R}^{3} \backslash\{0\}\right) C L^{2}\left(\mathbb{R}^{3}\right)$. These may be labelled by a parameter $L \in \mathbb{R}$, with $-\Delta^{L}$ corresponding to the boundary condition (replacing $(2.3))[(r \psi) /(\partial(r \psi) / \partial r)]{ }_{r}=0=L$ ( $r$ the radial coordinate). The value of $L$ for which $-\Delta^{L}$ well-approximates $-\Delta+\widetilde{V}$ ( $\widetilde{V}$ compactly supported, and, say spherically symmetric) is then the three dimensional scattering length $L$ of $V$. This is defined in the standard way (see e.g. [45]) by matching the unique smooth, spherically symmetric solution $\psi_{\text {true }}^{\text {static }}$ of $(-\Delta+V) \psi_{\text {true }}^{\text {stac }}=0$ outside the support of $V$ with the solution - replacing $(5.7)-r \psi_{L}$ $=$ const $(1+r / L))$. $L$ is thus easily seen to be given by the three-dimensional "fitting formula" (replacing (5.9))

$$
\left.L=\left[\left(r \psi_{\text {true }}^{\text {static }}\right) / \partial\left(r \psi_{\text {true }}^{\text {static }}\right) / \partial r\right)\right] \uparrow_{r=a}-a .
$$

21. A suitable precise statement would be e.g. to fit $R$ to the quantity

$$
2 e^{-c} \lim _{k \rightarrow 0} k^{-1} \exp ([\pi /(2 \tan (\gamma(k)))]),
$$


where $\gamma(p)$ is the change in the $(m=0)$ phase shift (cf. (2.14) and Note $\langle 14\rangle)$. (See [42] for further discussion and we recall that $R=0$ should be identified with $R=\infty$.)

22. We caution against a number of incorrect "derivations" of this result. (The "derivations" of $R=0$ which are criticized below would apply equally well in the case of a purely rounded-off cone or in the case of a rounded-off cone with a strong non-negative potential $\widetilde{V}$. As we discuss in Sect. 5.2, in the latter case the correct $R$-value often differs importantly from zero, and hence these "derivations" must be incorrect - and the fact that they yield the correct answer in the case of a purely rounded-off cone must be regarded as fortuitous!)

Pitfall 1. One might be tempted to argue that, since in the "true" problem, the wave-function is "regular" everywhere, it must not diverge at $\varrho=0$ in the corresponding idealized problem and hence that the appropriate $R$-value is zero. However, this must be an incorrect argument since one could similarly argue that for any smooth potential the correct $R$-value is zero. Yet, for any given fixed support, one can easily find potentials with any given scattering length $R$, and hence any given fitted $R$ value.

Pitfall 2. One can show (cf. [42] and [43]) that for all sequences of rounded-off cones with roundedness support which tends to zero, and for many sequences of potentials (and, indeed, for all sequences of non-negative potentials) $V$ with support which decreases to zero, the dynamics of Eq. (5.4) will tend, in a suitable sense, to the $R=0$ dynamics on an idealized cone. It is, however, incorrect to conclude from this that, for a "true" cone of "small" roundedness support $a$, and, say, a nonpositive potential with the same small support, the $R=0$ idealized dynamics should well-approximate the true dynamics at scales much larger than $a$. The reason is that, as explained in Sect. 5.2, in two dimensions, a potential of small support can still have a reasonably large effect at "medium scales" because of the logarithmic fall-off of "effect" with scale in two dimensions mentioned in the text (see also [42]). As far as effects at such scales are concerned, one must regard such a sequence as still far from convergence until the radius of the support is exponentially smaller than $a$.

Pitfall 3. One might argue that, since (for any metric $\tilde{h}$ and any non-positive potential $\tilde{V}$ ) the spectrum of minus the true Laplacian is positive (and, in particular, has no bound states) then, it must be approximated by the $R=0$ extension of minus the idealized Laplacian, since all the other extensions have a "bound state" (as we saw in Sect. 1). Such an argument must be incorrect because one could similarly argue for $R=0$ in the case of a strong positive potential of small support in flat spacetime, whereas a better approximation would be obtained at large scales by the exact solution to the fitting formula (5.9). The point is that, as explained in Sect. 5.2 (see also [42]), the bound state in the resulting idealized dynamics has to do with small length scales where one does not expect the approximation to be believable.

23. In Eq. (43) of [52], the local mass term $\tilde{V}$ is replaced by some multiple of a 2-dimensional delta function potential, $q^{2} \delta^{2}(x) / \pi$. We wish to emphasize that the latter is not a mathematically well-defined notion, and, as a result, it makes no physical sense to assign any particular finite value to the quantity $q^{2} / \pi$. In fact, as emerges in [52], it must be subject to an infinite renormalization (cf. the discussion in [28].) Comparison of the present discussion with the discussion around Eq. (43) 
in [52] makes clear: (1) Heuristically, the non-trivial self-adjoint extension $-\Delta^{R}$ is the same thing as $-\Delta$ plus a (renormalized) delta function potential. (2) Working from the outset in terms of $-\Delta^{R}$ has the advantage that it bypasses any need for infinite renormalizations. Ultimately, this point of view is both mathematically better defined and simpler. It is also more "physical" since the parameter $R$ (in contrast to $q^{2} / \pi$ ) is a well-defined physically meaningful quantity - the scattering length as we define it here - which is calculable in terms of the true local mass function $\widetilde{V}$ by the fitting formula (5.9). In relating the present discussion with the discussion around Eq. (43) in [52], one should (as we explain in the text) identify the scattering length $R$ with $2 e^{-c} \Lambda^{-1}$, where $\Lambda$ is the "cut-off that depends on the structure of the string" of [52] (see after Eq. (47) there).

We remark that the assumption of a strong local mass term is justified, e.g. in the case of a bosonic superconductor - by the argument in [52] that (in the notation of [52]) $K e^{2} / \pi \gtrsim 1$. Our fitting formula and the above relation between $R$ and $\Lambda$ then justifies the remark in [52] that "an extreme upper bound on $\Lambda$ might be $\Lambda=10^{19} \mathrm{GeV}$ (if the string thickness is the Planck length)." Note however, that, e.g. by Eq. (5.10), a weak local mass term could easily lead to values for $\Lambda$ exponentially larger than the Planck energy.

Acknowledgements. This work was supported in part by the Schweizerischer Nationalfonds. B.S.K. thanks the SERC for the award of an advanced fellowship.

\section{References}

1. Vilenkin, A.: In: 300 Years of Gravity. Hawking, S.W., Israel, W. (eds.). Cambridge: Cambridge University Press 1987

2. Aryal, M., Ford, L.H., Vilenkin, A.: Phys. Rev. D 34, 2263 (1986)

3. Linet, B.: J. Gen. Rel. Grav. 17, 1109 (1985)

4. Smith, A.G.: Gravitational effects of an infinite straight cosmic string on classical and quantum fields: self-forces and vacuum fluctuations. Tufts University Preprint, June 1986

5. Helliwell, T.M., Konkowski, D.A.: Phys. Rev. D 34, 1918 (1986)

6. Linet, B.: Phys. Rev. D 35, 536 (1987)

7. Frolov, V.P., Serebriany, E.M.: Phys. Rev. D 35, 3779 (1987)

8. Dowker, J.S.: Phys. Rev. D 36, 3095 (1987)

9. Dowker, J.S.: Class. Quantum Grav. 4, L 157 (1987)

10. Davies, P.C.W., Sahni, V.: Class. Quantum Grav. 5, 1 (1988)

11. De Witt, B.S.: Phys. Rep. 19, 295 (1975)

12. Kay, B.S.: Phys. Rev. D 20, 3052 (1979)

13. 't Hooft, G.: Commun. Math. Phys. 117, 685 (1988)

14. Deser, S., Jackiw, R.: Commun. Math. Phys. 118, 495 (1988)

15. de Barros Cobra Damgaard, B., Roemer, H.: Lett. Math. Phys. 13, 189 (1987)

16. Misner, C.W., Thorne, K.S., Wheeler, J.A.: Gravitation. San Francisco: Freeman 1973

17. Reed, M., Simon, B.: Methods of modern mathematical physics. Vol. I. Functional analysis. New York, London: Academic Press 1972

18. Reed, M., Simon, B.: Methods of modern mathematical physics. Vol. II. Fourier analysis, selfadjointness. New York, London: Academic Press 1975

19. Stakgold, I.: Green's functions and boundary value problems. New York: Wiley 1979

20. Wald, R.M.: J. Math. Phys. 20, 1056 (1979); Erratum, J. Math. Phys. 21, 218 (1980)

21. Kay, B.S., Wald, R.M.: Class. Quantum Grav. 4, 893 (1987)

22. Kay, B.S., Wald, R.M.: Theorems on the uniqueness and thermal properties of stationary, nonsingular, quasi-free states on spacetime with a bifurcate Killing horizon. Phys. Rep., in press, 1991

23. Segal, I.E.: Mathematical problems of relativistic physics. Providence: American Mathematical Society 1963 
24. Kay, B.S.: Commun. Math. Phys. 62, 55 (1978)

25. Studer, U.M.: Quantum mechanics and quantum field theory on spacetimes with conical singularities. Diploma Thesis, ETH Zürich 1988

26. Chernoff, P.R.: J. Funct. Anal. 12, 401 (1973)

27. Coddington, E.A., Levinson, N.: Theory of ordinary differential equations. New York: McGraw-Hill 1955

28. Albeverio, S., Gesztesy, F., Hoegh-Krohn, R., Holden, H.: Solvable models in quantum mechanics. Berlin, Heidelberg, New York: Springer 1988

29. Linet, B.: Phys. Rev. D 33, 1833 (1986)

30. Kay, B.S.: Springer Lect. Notes in Math., Vol. 905, p. 272. Berlin, Heidelberg, New York: Springer 1982

31. Dowker, J.S.: J. Phys. A 10, 115 (1977)

32. Gradshteyn, I.S., Ryzhik, I.M.: Table of integrals, series, and products. New York, London: Academic Press 1980

33. de Sousa Gerbert, P., Jackiw, R.: Commun. Math. Phys. 124, 229 (1989)

34. Dimock, J., Kay, B.S.: Ann. Phys. (NY) 175, 366 (1987)

35. Gott, J.R.: Ap. J. 288, 422 (1985)

36. Hiscock, W.A.: Phys. Rev. D 31, 3288 (1985)

37. Frolov, V.P., Israel, W., Unruh, W.G.: Phys. Rev. D 39, 1084 (1989)

38. Hiscock, W.A.: Phys. Lett. B 188, 317 (1987)

39. Reed, M., Simon, B.: Methods of modern mathematical physics, Vol. III. Scattering Theory. New York, London: Academic Press 1979

40. Newton, R.G.: Scattering theory of waves and particles. New York: McGraw-Hill 1966

41. Gibbons, G.W., Ruiz Ruiz, F., Vachaspati, T.: Commun. Math. Phys. 127, 295-312 (1990)

42. Kay, B.S.: When can a small object have a big effect at large scales?, in preparation

43. Kay, B.S.: Theorems on sequences of Schrödinger potentials with supports which decrease to zero, in preparation

44. Alford, M.G., March-Russell, J., Wilczek, F.: Nucl. Phys. B 328, 140 (1989)

45. Blatt, J.M., Weisskopf, V.F.: Theoretical nuclear physics. New York: Wiley (London: Chapman and Hall) 1952

46. Bollé, D., Gesztesy, F.: Phys. Rev. Lett. 52, 1469 (1984)

47. Bollé, D., Gesztesy, F.: Phys. Rev. A 30, 1279 (1984)

48. Gregory, R.: Phys. Rev. Lett. 59, 740 (1987)

49. Landsman, K.: Quantization and superselection sectors II: Dirac monopole and AharonovBohm effect, Rev. Math. Phys. 2,73 (1990)

50. Aharonov, Y., Bohm, D.: Phys. Rev. 119, 485 (1959)

51. Everett, A.E.: Phys. Rev. D 24, 858 (1981)

52. Witten, E.: Nucl. Phys. B 249, 557 (1985)

53. Kay, B.S.: On the electromagnetic self-force due to a superconducting cosmic string, in preparation

54. Vilenkin, A.: Phys. Rev. D 23, 852 (1981)

55. Garfinkle, D.: Phys. Rev. D 32, 1323 (1985)

56. Everett, A.E.: Phys. Rev. D 61, 1807 (1988)

57. Preskill, J.: Vortices and Monopoles. In: Architecture of the fundamental interactions at short distances. Ramond, R., Stora, R. (eds.). Amsterdam: North Holland 1987

58. Allen, B., Ottewill, A.: Effects of curvature couplings for quantum fields on cosmic string space-times. Phys. Rev. D 42, 2669 (1990)

59. Exner, P., Šeba, P. (eds.): Application of self-adjoint extensions in quantum physics. (Proceedings, Dubna.) Lect. Notes in Phys., Vol. 324. Berlin, Heidelberg, New York: Springer 1989

60. Emmrich, C., Römer, H.: Commun. Math. Phys. 129, 69 (1990)

61. de Sousa Gerbert, Ph.: Phys. Rev. D 40, 1346 (1989)

62. Perkins, W.B., Davis, A.-C.: Nucl. Phys. B 349, 207 (1991)

63. Gregory, R., Perkins, W.B., Davis, A.-C., Brandenberger, R.H.: Cosmic Strings and Baryon Decay Catalysis. In: The Structure and Evolution of Cosmic Strings. Gibbons, G.W., Hawking, S.W., Vachaspati, T. (eds.) Cambridge, Cambridge University Press 1990 
